\title{
Auswirkungen von Teleheimarbeit auf geschlechtsspezifische Einkommensungleichheiten in Arbeitsorganisationen
}

Die Bedeutung unterschiedlicher Umsetzungsformen

\author{
Anja-Kristin Abendroth $\cdot$ Martin Diewald
}

Zusammenfassung Teleheimarbeit wird vielfach als eine Lösung für Vereinbarkeitsprobleme zwischen Beruf und Privatem diskutiert. Inwiefern Teleheimarbeit auch dazu beiträgt, geschlechtsspezifische Einkommensungleichheiten abzubauen, ist jedoch bisher unklar. Im Rahmen dieses Beitrages argumentieren wir, dass Teleheimarbeit in Betrieben sowohl dazu beitragen kann, diese abzubauen, als auch, sie zu verstärken. Entscheidend dafür ist die konkrete Umsetzung von Teleheimarbeit in den jeweiligen Betrieben, die unterschiedlichen Normen folgt. Wir differenzieren die Umsetzung von Teleheimarbeit (a) als Implementationslücke, (b) als Stigmatisierung, (c) als Entgrenzung und (d) als Unterstützung. Die Ergebnisse auf Basis von organisationalen Fixed-effects-Modellen für eine repräsentative Stichprobe von Beschäftigten in deutschen Großbetrieben zeigen eine beträchtliche betriebliche Heterogenität hinsichtlich sowohl dieser Umsetzungsformen als auch im Ausmaß des „gender wage gap“. Nur eine Umsetzung von Teleheimarbeit, die mit einer Unterstützung der Vereinbarkeit von Beruf und Familie durch Vorgesetzte einhergeht, kann Einkommensunterschiede zwischen den Geschlechtern verringern. Geht Teleheimarbeit als Entgrenzung mit Erwartungen an Mehrarbeit einher, werden dagegen Einkommensunterschiede zwischen den Geschlechtern sogar akzentuiert.

Schlüsselwörter Teleheimarbeit · Einkommensungleichheit ·

Geschlechterungleichheit · Vereinbarkeit · Mehrebenendesign · Organisationale Ungleichheit 


\title{
Consequences of Home-based Telework on the Gender Pay Gap in Work Organizations
}

The Relevance of Different Forms of Implementation

\begin{abstract}
Home-based telework is increasingly discussed as a solution for problems in reconciling demands in work and personal life. To what extent home-based telework can also contribute to reduce the gender wage gap is unclear until now. We argue that home-based telework can contribute to reducing the gender pay gap but can also increase it. Important is how home-based telework is implemented in workplaces depending on existing norms of the ideal worker. We differentiate the implementation of home-based telework as (a) incomplete implementation, (b) as stigmatization, (c) as blurred boundaries, and (d) as supportive. The results of organizational fixed-effect models based on a representative sample of employees in large workplaces in Germany show relatively high workplace heterogeneity in the forms of implementation as well as in the gender pay gap. Only an implementation which involves support for work-life balance by supervisors contributes to a reduction in the gender wage gap. If the use of home-based telework in workplaces goes along with high expectations on overwork, blurring the boundaries between the life domains, the gender pay gap is reinforced.
\end{abstract}

Keywords Home-based telework · Telecommuting · Working from home · Home office $\cdot$ Gender pay gap · Organizational inequality · Employer-employee study · Work-life integration

\section{Einleitung}

Die informationstechnische Vernetzung hat zunehmend Teleheimarbeit als Arbeitsform in räumlicher Distanz zum Betrieb ermöglicht. Darunter verstehen wir im Folgenden die Möglichkeit, als betrieblich Beschäftigte Arbeit ganz oder teilweise zuhause erledigen zu können (Kleemann 2005), wobei wir keine Einschränkung vornehmen, wie groß oder klein dieser Anteil ist, und ob es sich dabei um formalisierte oder informelle Arrangements handelt. Nicht mit eingeschlossen sind dagegen mobile Arbeitsformen, die außerhalb des Betriebs ausgeführt werden, weil es die Arbeitstätigkeit verlangt, wie z. B. Kundenbetreuung oder Dienstreisen. Vor dem Hintergrund neuerer Entwicklungen in digitalen Technologien (z. B. digitale Netzwerke, digitale Informationssysteme, Data Clouds, digitale Steuerungsmöglichkeiten von Maschinen und Arbeitsprozessen) ist Teleheimarbeit für immer mehr Beschäftige realisierbar (Kossek 2016). Tatsächlich zeigt der für deutsche Großbetriebe repräsentative LEEP-B3 Datensatz aus dem Jahr 2012/13 mit mehr als 6000 Beschäftigten in 100 Betrieben (Abendroth et al. 2014; Diewald et al. 2015; Pausch et al. 2013), dass es in fast jedem Betrieb in gewissem Umfang, formell oder informell, Teleheimarbeit gibt $^{1}$.

\footnotetext{
${ }^{1}$ Nur in einem von hundert Betrieben wurde weder durch den Betrieb noch durch Beschäftigte angegeben, dass es Teleheimarbeit im Betrieb oder der Dienststelle gibt.
} 
Im öffentlichen und politischen Diskurs wird Teleheimarbeit zunächst als Arbeitsarrangement für eine bessere Vereinbarkeit von Beruf und Privatem diskutiert. Dabei sind die Einschätzungen der Wirkung von Teleheimarbeit durchaus kontrovers. Zum einen wird darauf verwiesen, dass sich mehr Beschäftigte die Möglichkeit der Teleheimarbeit für eine flexiblere Anpassung der Anforderungen aus verschiedenen Lebensbereichen wünschen (Brenke 2016). Damit einhergehend wird vom Bundesarbeitsministerium ${ }^{2}$ sogar gefordert, ein Recht auf Teleheimarbeit, auch häufig als Homeoffice tituliert, gesetzlich zu verankern. Zum anderen zeigen wissenschaftliche Untersuchungen, dass Teleheimarbeit statt mit geringeren sogar oft mit größeren Problemen der Vereinbarkeit von Beruf und Privatem und einer Intensivierung von Arbeit einhergeht (Allen et al. 2015; Byron 2005; Chung und Van der Lippe 2018; Gajendran und Harrison 2007; Kossek et al. 2010; Lott und Chung 2016).

Teleheimarbeit wird auch als Chance wahrgenommen, Einkommensungleichheiten zwischen den Geschlechtern abzubauen (Konrad und Yang 2012; Van der Lippe et al. 2018), die unter anderem auf die geschlechtsspezifische Arbeitsteilung und damit verbundene spezifische Vereinbarkeitsprobleme sowie bewusste und unbewusste Stereotypisierungen gegenüber Frauen zurückgeführt werden, in denen sie als weniger produktiv gelten (z. B. Achatz 2005; Ridgeway und Correll 2004). Die Frage nach den Konsequenzen von Teleheimarbeit für geschlechtsspezifische Einkommensungleichheiten steht im Fokus des vorliegenden Beitrags, wobei es hier ausschließlich um bereinigte Einkommensungleichheiten gehen soll. Die entscheidende Frage ist, ob das Angebot von Teleheimarbeit in Betrieben solchen Stereotypisierungen von Frauen, insbesondere Müttern, entgegenwirkt oder sie sogar noch verstärkt. Im ersten Fall bedeutet Teleheimarbeit eine Investition in Beschäftigte mit familiären Verpflichtungen, im zweiten Fall bedeutet Teleheimarbeit neue Möglichkeiten der Grenzziehungen zwischen angenommenen karriereorientierten und familienorientierten Beschäftigten entlang der Merkmale Geschlecht und Elternschaft. Die Nutzung von Teleheimarbeit geht dann mit Stereotypisierungen einher, weniger beruflich engagiert zu sein, und führt so zu schlechteren monetären Gratifikationschancen (Almer et al. 2004; Blair-Loy und Wharton 2002). Diese Stereotypisierungen gelten eher für Frauen und insbesondere Mütter, die Teleheimarbeit häufiger für eine bessere Vereinbarkeit von Beruf und Privatem nutzen, wohingegen Männer mit Teleheimarbeit eher versuchen, hohen Arbeitsanforderungen an Mehrarbeit, Erreichbarkeit und Belastungsfähigkeit gerecht zu werden (vgl. Lott 2018).

Bisher vorliegende Forschungsergebnisse zu den Implikationen von Teleheimarbeit für geschlechtsspezifische Einkommensungleichheiten sind nicht eindeutig. Die Nutzung von flexiblen Arbeitsplatzarrangements wie Teleheimarbeit förderte in manchen Studien positive Einkommensentwicklungen (Konrad und Yang 2012; Lott und Chung 2016) und reduzierte die geschlechtsspezifischen Einkommensungleichheiten (Van der Lippe et al. 2018), in anderen trug sie umgekehrt zu Karriereeinbußen bei (Almer et al. 2004; Glass 2004) oder sie wirkte sich für Männer positiv und für Frauen negativ auf das Einkommen aus (Lott und Chung 2016). Für

${ }^{2}$ https://www.golem.de/news/koalitionsvertrag-bundesarbeitsminister-will-recht-auf-homeofficedurchsetzen-1903-139782.html (Zugegriffen: 04.03.2019). 
Deutschland zeigt Lott (2015), dass Flexibilität häufig im Interesse des Arbeitgebers genutzt wird. Wann Teleheimarbeit als betriebliche Vereinbarkeitsmaßnahme hilft, Einkommensunterschiede zwischen Männern und Frauen innerhalb von Betrieben anzugleichen und wann Teleheimarbeit eher Einkommensungleichheiten verstärkt, ist weder theoretisch eindeutig noch empirisch hinreichend geklärt.

Aufbauend auf der Theorie organisationaler oder relationaler Ungleichheiten (Tomaskovic-Devey und Avent-Holt 2019; Tomaskovic-Devey 2014) ist es Ziel unseres Beitrags zu untersuchen, inwiefern die Umsetzung von Teleheimarbeit zwischen Betrieben variiert und inwiefern sich daraus unterschiedliche Konsequenzen für geschlechtsspezifische Einkommensungleichheiten innerhalb von Betrieben ergeben. Einerseits können der Umsetzung von Teleheimarbeit in Betrieben jeweils unterschiedlichen Betriebskulturen zugrunde liegen, die der Norm des ,idealen Beschäftigten“ entsprechen oder von ihr abweichen (Acker 2006; Chung 2017a, 2017b; Den Dulk 2001; Swanberg et al. 2005). Andererseits verweist der Neo-Institutionalismus darauf, dass es dabei auch zu an „Heuchelei“ (Brunsson 2003) grenzenden Widersprüchen zwischen betrieblicher Selbstdarstellung nach außen einerseits und betrieblicher Praxis in der Verfügbarkeit und Ausgestaltung von Teleheimarbeit für die Beschäftigten andererseits kommen kann (z. B. McDonald et al. 2005; Den Dulk und De Ruiter 2008). Wir greifen in unserem Beitrag beide Perspektiven auf und unterscheiden hinsichtlich der Umsetzung von Teleheimarbeit (a) eine mögliche Lücke zwischen formellem Angebot und tatsächlicher Verfügbarkeit für die Beschäftigten (z. B. Den Dulk und De Ruiter 2008; McDonald et al. 2005); (b) eine stigmatisierende Umsetzung, die mit der Wahrnehmung einhergeht, dass die Nutzung von Teleheimarbeit geringeres berufliches Engagement bedeutet (Acker 2006; Blair-Loy und Wharton 2002); (c) eine vereinbarkeitsfördernde Umsetzung, die sich als Teil familienfreundlicher Betriebskulturen durch eine Unterstützung durch die direkten Vorgesetzten auszeichnet (z. B. Den Dulk 2001; Kossek et al. 2010); sowie (d) eine mit Entgrenzung einhergehende Umsetzung, bei der die Nutzung von Teleheimarbeit mit hohen Erwartungen an Mehrarbeit einhergeht, wie es für anforderungsbetonte Betriebskulturen typisch ist (z. B. Cha und Weeden 2014; Lott und Chung 2016).

Mit diesem Ansatz tragen wir zum Stand der Forschung in folgender Hinsicht bei: Erstens betrachten wir die Relevanz einer unterschiedlichen Umsetzung von Teleheimarbeit für geschlechtsspezifische Einkommensungleichheiten innerhalb von Betrieben, was bisher durch das Fehlen von linked employer-employee-Daten mit umfassenden Informationen zu Arbeitgebern und Arbeiternehmern kaum möglich war, vor allem nicht in Deutschland. Eine Ausnahme ist die Studie von Van der Lippe et al. (2018), die für einen nicht repräsentativen europäischen Datensatz zeigt, dass Teleheimarbeit eher mit einer Reduzierung geschlechtsspezifischer Einkommensungleichheiten einhergeht. Analysen zeigen jedoch keinen Einfluss von Teleheimarbeit für Deutschland (Van der Lippe et al. 2018). Angesichts des mittlerweile stark verbreiteten Anbietens von Teleheimarbeit in größeren Betrieben fokussieren wir in diesem Beitrag nicht mehr auf das „Ob“ eines solchen Angebots, sondern auf das „Wie“. Insbesondere erlaubt hierbei die Nutzung des linked employer-employeeDatensatzes LEEP-B3 den Abgleich zwischen Betriebsangaben und Beschäftigtenangaben zur Verfügbarkeit und Nutzung von Teleheimarbeit. Zweitens folgen wir mit dem Fokus auf Einkommensungleichheiten innerhalb von Betrieben einem generell 
gestiegenen Forschungsinteresse an betrieblichen Ungleichheitsregimen als wichtigem Bestandteil der gesellschaftlichen Ungleichheitsstruktur. Wir beziehen uns hier auf geschlechtsspezifische Einkommensungleichheiten, für die Unterschiede an Humankapital, beruflichen und familiären Merkmalen, aber auch Belastungen wie Überstunden bereits berücksichtigt wurden, um Einflüsse der Umsetzung von Teleheimarbeit für geschlechtsspezifische Einkommensungleichheiten unabhängig von der Relevanz für berufliche Investitionschancen von Männern und Frauen zu untersuchen. Wir knüpfen damit, ausgehend von theoretisch-abstrakten Mechanismen, an Bestrebungen an, konkrete betriebliche Bedingungen zu identifizieren die solche Ungleichheiten hervorbringen (Diewald und Faist 2011). Drittens unterscheiden wir bei den Auswirkungen einer unterschiedlichen Umsetzung von Teleheimarbeit zwischen Männern und Frauen mit und ohne Kinder, denn wir gehen davon aus, dass Elternschaft bei beiden Geschlechtern unterschiedlich wahrgenommen und bewertet wird, wenn sie Teleheimarbeit in Anspruch nehmen. Wir leisten damit einen Beitrag dazu, wie zwei Heterogenitätsmerkmale, nämlich Geschlecht und Elternschaft, bei der Genese relationaler Ungleichheiten ineinandergreifen. Viertens ist bisher zwar thematisiert worden, dass Vereinbarkeitsmaßnahmen unterschiedlichen Rationalitäten folgen können, aber zu den Auswirkungen einer entsprechend unterschiedlichen Umsetzung von Teleheimarbeit für geschlechtsspezifische Einkommensungleichheiten innerhalb von Betrieben ist bisher für Deutschland nichts bekannt.

\section{Theoretische Ansätze: Verfügbarkeit von Teleheimarbeit und geschlechtsspezifische Einkommensungleichheiten in Betrieben}

Arbeitgeber entscheiden im Rahmen tariflicher Bindungen über die Höhe des Gehalts. Neben Qualifikationen und Anforderungen, wie dem akkumulierten Humankapital, Berufsmerkmalen und besonderen beruflichen Belastungen wie Überstunden, können auch Heterogenitäten, wie Geschlecht oder Elternschaft, eine Rolle spielen, und zwar auch dann, wenn für Unterschiede in Beruf, Qualifikationen und Anforderungen kontrolliert werden (Cha und Weeden 2014; Correll et al. 2007; England 2010). Solche sogenannten bereinigten Einkommensbenachteiligungen werden auf bewusste und unbewusste Stereotypen gegenüber Frauen und Müttern zurückgeführt, nach denen sie als weniger produktive Mitarbeiter gelten (Correll et al. 2007; England 1994, 2010; Oakley 2000; Phelps 1972; Reskin 2000; Ridgeway und Correll 2004). Geschlechtersoziologische Ansätze verweisen darauf, dass wir automatisch nach Geschlecht kategorisieren und dabei geschlechtsspezifische Aufgabenzuweisungen und angenommene geschlechtsspezifische Fähigkeiten und Statuswahrnehmungen, bewusst oder unbewusst, Bewertungen und Behandlungen prägen (vgl. Ridgeway und Correll 2004; Risman 2014). Solche Stereotypen treffen Mütter noch stärker als Frauen ohne Kinder (Ridgeway und Correll 2004). Auch Arbeitsorganisationen bestimmen mit, inwiefern diese kulturellen Vorstellungen von Geschlecht und damit verbundene Stereotypisierungen salient sind und damit Einkommenschancen von Männern und Frauen beeinflussen (Ridgeway und Correll 2004). Damit einhergehend verweist die relationale Theorie sozialer Ungleichheiten in Arbeitsorganisationen darauf, dass die Salienz solcher Stereotypisierungen im Betrieb auch 
beeinflusst, inwiefern Frauen und Männer selbst Forderungen bezüglich ihres Einkommens stellen, und inwiefern diese als legitim erachtet werden (Mechanismus des „,claims making“; Tomaskovic-Devey und Avent-Holt 2019). Zum anderen dominiert in vielen Betrieben als Norm des ,idealen Beschäftigten“ die Vorstellung von Arbeitskräften mit kontinuierlicher Vollzeitbeschäftigung und wenigen Verpflichtungen außerhalb der Arbeit, wie sie typisch männlichen Erwerbsverläufen entspricht. Da dies Frauen und insbesondere Müttern durch die hauptsächliche Übernahme der Haushalts- und Sorgearbeit deutlich schwerer fällt, bedeutet dies einen Mechanismus der „Chancenhortung “"3 , der hohe Einkommen eher für Männer zugänglich macht (Acker 2006; Hodges und Budig 2016; Kossek et al. 2010; Tomaskovic-Devey und Avent-Holt 2019). Die überproportionale Vergütung von Überstunden, die einen erheblichen Einfluss auf Einkommensunterschiede zwischen den Geschlechtern hat, weil hauptsächlich Männer diese leisten, zeigt, wie tief die geschlechtsspezifische Arbeitsteilung mit Leistungsmerkmalen verwoben ist (Cha und Weeden 2014).

Familienfreundliche Personalpolitiken, wie Teleheimarbeit hingegen, adressieren typisch weibliche Erwerbsverläufe mit familiären Verpflichtungen und Problemen der Vereinbarkeit von Beruf und Familie. Sie stehen damit eher nicht im Einklang mit der klassischen Norm des idealen Beschäftigten. Inwiefern Sie dazu beitragen, Einkommensungleichheiten zwischen den Geschlechtern zu verringern oder zu verstärken, ist unklar. Zum einen kann diese Investition in die Vereinbarkeit von Beruf und Privatem Stereotypen gegenüber Frauen abbauen, die sie als weniger produktiv darstellen. Ihnen wird damit nicht eine per se geringere Leistungsbereitschaft oder -fähigkeit unterstellt, sondern es wird die Organisation von Arbeit so gestaltet, dass sie umgekehrt deren bessere Entfaltung ermöglicht. Jedoch ist es umgekehrt auch möglich, dass eine Adressierung von familiären Verpflichtungen und Vereinbarkeitsproblematiken durch Teleheimarbeit die Kategorie Geschlecht und insbesondere Mutterschaft in Arbeitsorganisationen besonders salient macht. Teleheimarbeit stellt dann eher ein Angebot dar, auch mit eingeschränkter Leistungsbereitschaft aufgrund konkurrierender Verpflichtungen zu arbeiten, allerdings auf Arbeitsplätzen mit entsprechend geringerer Entlohnung (Chung 2017a, 2017b; Konrad und Yang 2012).

In welche Richtung die Umsetzung von Teleheimarbeit gedacht ist, durch welche Organisationskulturen sie geprägt ist und inwiefern sie klassischen Normungen eines idealen Arbeitnehmers folgen, darüber können unterschiedliche Formen der Umsetzungen Auskunft geben, die auf unterschiedliche Verteilungsmuster und Motive bei der Umsetzung von Teleheimarbeit verweisen (Chung 2017b; Den Dulk 2001; Swanberg et al. 2005). Betriebe unterscheiden sich in ihrer Historie, ihrer Zusammensetzung, Kultur oder ihren Umwelten, die unterschiedliche Ungleichheitsregime prägen und damit auch die Verhandlung der Umsetzung von Teleheimarbeit sowie Einkommensverhandlungen (Tomaskovic-Devey und Avent-Holt 2019). Im Folgenden differenzieren wir auf Basis bestehender Forschung (z. B. Chung 2017b; Den Dulk 2001; Swanberg et al. 2005) vier Umsetzungsmuster von Teleheimarbeit in

\footnotetext{
${ }^{3}$ Chancenhortung geht als Begriff auf Tilly (1998) zurück, der dies als einen zentralen Mechanismus der Ungleichheitsgenese ausgeführt hat und dabei wesentlich auf die Weber'sche Definition sozialer SchlieBung zurückgreift.
} 
Betrieben - Implementationslücke, Stigmatisierung, Entgrenzung und Unterstützung - und argumentieren, dass sich aus den unterschiedlichen Umsetzungsmustern unterschiedliche Konsequenzen für geschlechtsspezifische Einkommensungleichheiten ergeben.

\subsection{Die Implementationslücke}

Auf Basis des Neo-Institutionalismus wird argumentiert, dass Betriebe familienfreundliche Arbeitsplatzarrangements nicht notwendigerweise als Investition in die Beschäftigungsbeziehung anbieten, sondern um Legitimität von außen zu erhalten, z. B. von der Politik, den Medien, Kunden oder Interessensvertretern auf Arbeitgeber- und Arbeitnehmerseite (Den Dulk 2001). Dies geschieht, weil Teleheimarbeit von politischer Seite gefordert wird, die Betriebe aber oft der klassischen Definition des idealen Arbeitnehmers anhängen und von der Gleichwertigkeit der Produktivität bei Teleheimarbeit nicht überzeugt sind (z. B. Acker 2006; Blair-Loy und Wharton 2002). Evidenzen für diese Strategie werden in Befunden gesehen, nach denen gerade große Betriebe und Betriebe im Öffentlichen Sektor familienfreundliche Arbeitsplatzarrangements formal anbieten (Den Dulk 2001). Die Unterscheidung von „talk“ und ,action“ oder Formal- und Aktivitätsstruktur (Brunsson 2003), impliziert, dass sich Organisationen als familienfreundlich präsentieren, um Legitimität von außen zu erreichen. Sie passen jedoch ihre Praktiken nicht entsprechend an, sondern bleiben weiterhin klassischen Beschäftigungsmustern und damit verbundenen geschlechtsspezifischen Einkommensverteilungen und -verhandlungen verhaftet.

Empirische Ergebnisse belegen solche Unterschiede zwischen Selbstdarstellung und Praxis (z. B. Den Dulk und De Ruiter 2008). Ein Indiz dafür ist beispielsweise, dass im Betrieb kaum ein Beschäftigter Kenntnis über die Verfügbarkeit von bestimmten personalpolitischen Maßnahmen besitzt. In solchen Betrieben wird zwar die Verfügbarkeit von Teleheimarbeit nach außen dargestellt, aber intern im Unternehmen nicht überzeugend kommuniziert und umgesetzt. Bei Entscheidungen und Verhandlungen über das Einkommen der Beschäftigten werden bei weiblichem Geschlecht und insbesondere Mutterschaft regelhafte höhere familiäre Verpflichtungen als Probleme für die Arbeitsproduktivität angenommen. Darauf aufbauend formulieren wir folgende Hypothese:

H1 Implementationslücken-Hypothese: In Betrieben, in denen die Selbstdarstellung zur Verfügbarkeit von Teleheimarbeit nicht mit einer tatsächlichen Verfügbarkeit im Betrieb einhergeht (Implementationslücke), sind Einkommensbenachteiligungen von Frauen im Vergleich zu Männern größer als in Betrieben, in denen die Selbstdarstellung zur Verfügbarkeit von Teleheimarbeit mit einer tatsächlichen Verfügbarkeit im Betrieb einhergeht.

\subsection{Umsetzung als Stigmatisierung}

Die Norm des idealen Beschäftigten muss jedoch nicht notwendigerweise die Folge haben, dass Teleheimarbeit als Implementationslücke im Betrieb umgesetzt wird. Eine weitere mögliche Umsetzung von Teleheimarbeit im Betrieb im Sinne der „Norm 
des idealen Beschäftigten“ ist die der Stereotypisierung, für die der Begriff des „Flexibilitätsstigmas" steht (z. B. Chung und Van der Horst 2018; Williams et al. 2013). Werden flexible Arbeitsplatzarrangements wie Teleheimarbeit im Betrieb für familiäre Verpflichtungen genutzt, werden Beschäftigte als weniger beruflich engagiert angesehen. Dies legt nahe, dass im Sinne von „compensating differential“-Argumenten (Filer 1985) Einkommen einerseits und familienfreundliche Arbeitsplatzarrangements andererseits als substitutiv und gegenseitig kompensierende Arbeitsgratifikationen eingesetzt werden. Teleheimarbeit wird demnach als Alternative zu einer höheren Bezahlung für diejenigen angeboten, die nicht der Norm eines ,idealen“ Beschäftigten entsprechen oder dies auch nicht wollen. Entsprechend äußern Beschäftigte die Sorge, dass die Nutzung von flexiblen Arbeitsplatzarrangements, wie auch Teleheimarbeit, Karrierechancen limitiert, weil sie dann als weniger beruflich engagiert wahrgenommen werden (Konrad und Yang 2012; Pleck 1993). Almer et al. (2004) belegen in einem Vignettenexperiment, dass Beschäftigte, die flexible Arbeitsplatzarrangements wie Teleheimarbeit nutzten, tatsächlich als weniger geeignet für Beförderungen angesehen wurden. Zudem konnten Blair-Loy und Wharton (2002) zeigen, dass Beschäftigte eher familienfreundliche betriebliche Politiken nutzen, wenn der oder die Vorgesetze genug Einfluss und Macht besaß, um die Beschäftigten vor negativen Konsequenzen für die Karriere zu bewahren. Aufbauend auf Signaling-Theorien (Spence 1973) und Stigmatisierungstheorien (Goffman 1963) sollte die Nutzung von Teleheimarbeit im Betrieb bei Vergütungs- und Positionsentscheidungen insbesondere dann als Signal für geringeres berufliches Engagement und konkurrierende familiäre Verpflichtungen genutzt werden, wenn Informationen über die tatsächlichen Fähigkeiten und Motivationen von Beschäftigten nur begrenzt zur Verfügung stehen oder nicht eindeutig sind (Konrad und Yang 2012). So erlaubt die Umsetzung von Teleheimarbeit als alternative Gratifikation zur hohen Bezahlung neue Möglichkeiten der Grenzziehung zwischen eher familienorientierten und eher karriereorientieren Beschäftigten entlang des Geschlechts. Frauen und insbesondere Mütter wählen aufgrund familiärer Verpflichtungen Teleheimarbeit (Lott 2019), und Männer werden eher abgeschreckt, Teleheimarbeit rein für familiäre Verpflichtungen zu verwenden. Wenn Männer Teleheimarbeit nutzen, dann zur Arbeitsintensivierung (Lott 2019). Auch Chung und Van der Horst (2018) verweisen darauf, dass das Flexibilitätsstigma geschlechtsdifferenziert ist. Es impliziert, dass insbesondere Frauen, und noch ausgeprägter Mütter, weniger Forderungen bezüglich ihres Einkommens stellen, dass Einkommensforderungen von ihnen als weniger legitim wahrgenommen werden, weil höhereEinkommen als Alternative zu einer besseren Vereinbarkeit von Beruf und Familie gelten. Daraus lässt sich ableiten, dass in Betrieben, in denen Teleheimarbeit im Sinne eines Flexibilitätsstigmas umgesetzt wird, Einkommensbenachteiligungen von Frauen mit und ohne Kinder besonders groß sind:

H2 Stigmatisierungs-Hypothese: In Betrieben, in denen die Nutzung von Teleheimarbeit mit Stereotypisierungen als weniger beruflich engagiert einhergeht, sind Einkommensbenachteiligungen von Frauen im Vergleich zu Männern größer als in Betrieben, in denen die Nutzung von Teleheimarbeit nicht mit Stereotypisierungen als weniger beruflich engagiert einhergeht. 


\subsection{Umsetzung als Entgrenzung}

Alternativ kann Teleheimarbeit auch die klassische Norm des idealen Beschäftigten noch einmal zuspitzen. Sie wird dann als Mittel zur Entgrenzung von Arbeit eingesetzt (z. B. Abendroth und Reimann 2018; Gambles et al. 2006; Kelliher und Anderson 2010; Lott und Chung 2016). Dies geschieht vor allem in Betrieben mit einer Organisationskultur, die hohe Belastungen und hohe Erwartungen an Mehrarbeit herausstellt. Gerade hochqualifizierte Beschäftigte nutzen flexible Arbeitsplatzarrangements wie Teleheimarbeit in diesem Sinne. Die Vereinbarkeit von Beruf und Familie mit hohen familiären Verpflichtungen wird dann durch Teleheimarbeit gerade nicht gefördert (Chung 2017b; Deitch und Huffman 2001; Glass 2004; Swanberg et al. 2005). In der Literatur wird hier auch auf das Autonomie-Paradox verwiesen, nach dem Beschäftigte, die örtlich und zeitlich flexibel arbeiten, auch mehr und härter arbeiten (Chung und Van der Horst 2018). So zeigen Abendroth und Reimann (2018) für deutsche Mittel- und Großbetriebe, dass die Nutzung von Teleheimarbeit tatsächlich eher mit größeren Konflikten zwischen Arbeit und Familie verbunden ist. Dies geschieht insbesondere durch damit einhergehende Überstunden und im Zusammenhang mit einer wahrgenommenen Organisationskultur, die sich durch Erwartungen an Belastungsfähigkeit und Mehrarbeit auszeichnet. Somit ist mit der Verfügbarkeit von Teleheimarbeit im Betrieb weder eine verstärkte Stereotypisierung von Nutzern als weniger karriereorientiert noch eine verstärkte Unterstützung von Frauen mit hohen familiären Verpflichtungen verbunden. Vielmehr wird Teleheimarbeit in einer Weise umgesetzt, die bei familiären Verpflichtungen kaum realisierbar ist. In ähnlicher Weise zeigen Van der Lippe und Lippényi (2018) mit einem europäischen Datensatz, dass Teleheimarbeit mit mehr Konflikten zwischen den Lebensbereichen einhergeht, wenn in Betrieben die klassische Norm des „,idealen“ Beschäftigten dominiert. Folgende Hypothese lässt sich ableiten:

H3 Entgrenzungs-Hypothese: In Betrieben, in denen die Nutzung von Teleheimarbeit mit einer hohen Erwartung an Mehrarbeit einhergeht, sind Einkommensbenachteiligungen von Frauen im Vergleich zu Männern größer als in Betrieben, in denen diese Erwartungen an Mehrarbeit nicht vorhanden sind.

\subsection{Umsetzung als Unterstützung}

Tauschtheoretische Konzepte der Beschäftigungsbeziehung (Coyle-Shapiro und Conway 2004; Cropanzano und Mitchell 2005) und „business-case“-Ansätze (Den Dulk et al. 2012) legen nahe, dass sich manche Betriebe einen ökonomischen Nutzen von der Investition in familienfreundliche Arbeitsplatzarrangements wie Teleheimarbeit versprechen. Teleheimarbeit als Teil familienfreundlicher Personalpolitik ist dann eine Investition in die Beschäftigungsfähigkeit von Mitarbeitern mit hohen familiären Verpflichtungen. Eine solche Umsetzung setzt familienfreundliche Betriebskulturen mit Unterstützung der Vorgesetzten für die Vereinbarkeit von Beruf und Privatem voraus (Den Dulk 2001; Kossek et al. 2010; Van der Lippe und Lippényi 2018). Der ökonomische Nutzen von Teleheimarbeit als Teil familienfreundlicher Personalpolitik für den Betrieb wird darin gesehen, eine flexible 
Anpassung von Beruf und Familie zu ermöglichen. Diese erlaubt es den Beschäftigten, trotz familiärer Verpflichtungen Zeit und Energie in die Arbeit zu investieren. Eine Stereotypisierung von Frauen mit und ohne Kinder, die sie als weniger oder zukünftig weniger produktiv ansieht, spielt hier allenfalls eine geringe Rolle. Vielmehr wird in sie investiert, damit ihre Beschäftigungsfähigkeit und Produktivität trotz familiärer Verpflichtungen erhalten bleibt. Eine Chancenhortung von Männern und Nicht-Eltern bei Einkommensverhandlungen wird damit erschwert. Die Nutzung von Teleheimarbeit signalisiert kein niedriges, sondern ein hohes Commitment, es ist eine Möglichkeit, sogar mehr Zeit und Energie in die Arbeit zu investieren, als wenn es diese Möglichkeit nicht gäbe (z. B. Abendroth und Den Dulk 2011; Byron 2005; Demerouti et al. 2001; Schaufeli und Bakker 2004). Je häufiger eine derart motivierte Nutzung von Teleheimarbeit in einem Betrieb ist, desto weniger sollten Stigmatisierungen von Personen mit familiären Verpflichtungen auftreten,

z. B. durch die Erfahrungen, die ein Vorgesetzter macht, und die er mit anderen Vorgesetzten teilt. Solche Erfahrungen werden durch bisherige Untersuchungen insofern bestätigt, als Beschäftigte mit flexiblen Arbeitsplatzregelungen wie Teleheimarbeit ähnliche oder sogar höhere Leistung und ein stärkeres Commitment im Vergleich zu ihren Kollegen zeigen (Baltes et al. 1999; Gajendran und Harrison 2007). Zudem ist zu erwarten, dass in diesem Fall auch Männer Teleheimarbeit für familiäre Verpflichtungen statt Entgrenzung nutzen. Daraus lässt sich ableiten, dass in Betrieben, in denen Teleheimarbeit mit einer Unterstützung für die Vereinbarkeit von Beruf und Privatem einhergeht, Einkommensnachteile von Frauen mit und ohne Kinder kleiner sind.

H4 Unterstützungs-Hypothese: In Betrieben, in denen die Nutzung von Teleheimarbeit mit einer Kultur der Unterstützung von Arbeit und Familie einhergeht, sind Einkommensbenachteiligungen von Frauen im Vergleich zu Männern kleiner als in Betrieben, die sich nicht durch familienfreundliche Unterstützungskulturen auszeichnen.

\section{Daten und Methoden}

\subsection{Daten}

Datengrundlage für die empirische Prüfung der formulierten Hypothesen ist der verknüpfte Arbeitnehmer-Arbeitgeber-Datensatz LEEP-B3 ${ }^{4}$, der im Projekt „Wechselwirkungen zwischen Verwirklichungschancen im Berufs- und Privatleben: Eine Untersuchung von Beschäftigten in unterschiedlichen Arbeitsorganisationen“ des Sonderforschungsbereiches 882 „Von Heterogenitäten zu Ungleichheiten“ an der Universität Bielefeld erhoben wurde (Abendroth et al. 2014; Diewald et al. 2015;

\footnotetext{
${ }^{4}$ https://doi.org/10.4119/unibi/sfb882.2014.12
} 
Pausch et al. 2013). Interviews mit Arbeitgebern ${ }^{5}$ und Arbeitnehmern ${ }^{6}$ in den Jahren 2012 und 2013 bieten umfassende Informationen sowohl über die Merkmale von Arbeitsorganisationen als Akteure und Opportunitätsstruktur als auch über die Arbeits- und Familiensituation von Beschäftigten in diesen Arbeitsorganisationen.

Der Datensatz umfasst 6454 Beschäftigte in 100 großen Arbeitsorganisationen aus verschiedenen Wirtschaftsbereichen in der Privatwirtschaft und dem Öffentlichen Dienst. Da die Stichprobe der Betriebe und Beschäftigten auf Basis von administrativen Daten zufällig gezogen wurde (Datenquelle: IAB Beschäftigtenhistorik $(\mathrm{BeH})$, Meldungen zur Sozialversicherung), ist es möglich, die realisierte Stichprobe mit der gesamten Arbeitspopulation aus Großbetrieben zu vergleichen. Selektivitätsanalysen für die Betriebs- und Beschäftigtenbefragung haben gezeigt, dass der LEEPB3 Datensatz in vieler Hinsicht repräsentativ für große Arbeitsorganisationen insgesamt ist. Zum Beispiel ist der Anteil von interviewten Frauen vergleichbar mit dem Anteil von Frauen in der Grundgesamtheit von Beschäftigten in Großbetrieben in Deutschland. Zudem sind keine signifikanten Unterschiede bezüglich Alter, Arbeitserfahrung, Betriebszugehörigkeit oder Einkommen festzustellen. Nur Beschäftigte ohne deutsche Nationalität sind unterrepräsentiert, ebenso wie Beschäftigte ohne Ausbildung und solche, für die der Bildungsgrad nicht bekannt ist. Weitere Informationen zu den Selektivitätsanalysen bieten die Methodenberichte zur Erhebung (Abendroth et al. 2014; Pausch et al. 2013). Die Erhebungsdaten wurden durch administrative Informationen des Instituts für Arbeits- und Berufsforschung (IAB) ergänzt. 5652 Beschäftigte aus der Befragung akzeptierten die Verlinkung mit administrativen Daten des IAB, die für das Ziel dieses Beitrags notwendig ist. Zur Beantwortung der Forschungsfrage haben wir eine Stichprobe von Arbeitsorganisationen gebildet, in der Männer sowie Frauen interviewt wurden, um überhaupt innerbetriebliche Einkommensungleichheiten zwischen Männern und Frauen untersuchen zu können. Ein Betrieb, in dem nur Männer und ein Betrieb, in dem nur Frauen befragt wurden, konnten daher für die Analysen nicht berücksichtigt werden. Dies führte zu einem Sample von 98 Großbetrieben und 5594 Beschäftigten. Beschäftigte ohne gültige Angaben zu den abhängigen oder unabhängigen Variablen sowie wenige Ausreißer wurden von der Analyse ausgeschlossen. Da nur in einem Betrieb weder auf Betriebs- noch auf Beschäftigtenebene die Verfügbarkeit von Teleheimarbeit angegeben wurde, wurde dieser aufgrund einer zu geringer Fallzahl für aussagekräftige Analysen ausgeschlossen. Das finale Sample für die Analyse beinhaltet 5099 Beschäftigte in 97 Großbetrieben. Durchschnittlich sind im finalen Sample 52,5 Beschäftigte pro Betrieb enthalten. ${ }^{7}$

\footnotetext{
${ }_{5}^{5}$ Die Betriebsbefragung des LEEP-B3 Daten wurden von 10 Mitarbeitern des IABs durchgeführt, die Projektmitarbeiter in den Stützpunktagenturen (ProIAB). In den meisten Fällen waren die Ansprechpartner der ProIABs in den Betrieben Personalverantwortliche (vgl. Pausch et al. 2013).

${ }^{6}$ Die Beschäftigtenbefragung wurde durch das Umfrageinstituts SOKO als Telefonbefragung außerhalb der Arbeitszeit durchgeführt (vgl. Abendroth et al. 2014).

${ }^{7}$ Minimalwert: 18 Beschäftigte; Maximalwert: 76 Beschäftigte.
} 


\subsection{Messinstrumente}

Abhängige Variable Für die abhängige Variable Stundenlohn wurde das monatliche Bruttoeinkommen inklusive flexibler, leistungs- oder erfolgsabhängiger Prämien in ein wochenbezogenes Einkommen umgerechnet und durch die vertraglichen Arbeitsstunden pro Woche geteilt. Anschließend wurde der natürliche Logarithmus gebildet. Im Fragebogen konnten Beschäftigte entweder ihr jährliches oder ihr monatliches Einkommen angeben ${ }^{8}$. Bei Angabe des Jahreseinkommens wurde dieses vorab durch 12 geteilt. Wenn die Teilnehmer der Befragung das spezifische Bruttoeinkommen nicht angeben wollten ( $8 \%$ des Beschäftigtensamples), wurden sie gefragt, welche der folgenden Gruppierungen am besten das persönliche Einkommen wiedergibt. Zur Auswahl standen: ,weniger als $500 €^{\star}$, ,,500 bis unter $1000 €$ “,

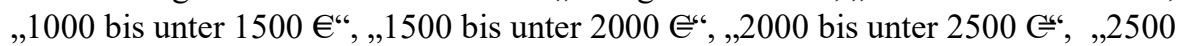
bis unter $3000 €$ “", „3000 bis unter $4000 €$ ", „4000 bis unter $5000 €$ “, ,5000 bis unter $7500 €$ “", ,7500 bis unter $10.000 €$ “" und „10.000€ oder mehr“. Für die Analyse wurde der Mittelwert jeder Gruppe verwendet. Der untersten Einkommensklasse wurde der Wert 500 zugeteilt, der obersten Klasse der Wert 10.000€. 188 Beschäftigte gaben keine Auskunft zu ihrem Einkommen. Einkommen von $0 €$ wurden in den Analysen nicht berücksichtigt; dies traf lediglich auf eine Person zu.

Unabhängige Variablen Die Variable Geschlecht-Familien-Kombination unterscheidet zwischen Frauen und Männern mit und ohne Kinder. Frauen mit Kindern wurden als Referenzkategorie gewählt. Um die Familiensituation genauer abzubilden, wurde das Alter des jüngsten Kindes verwendet. Für Männer und Frauen ohne Kinder wurde der Mittelwert vergeben. Die Beziehungssituation bildet ab, ob die jeweiligen Beschäftigten (0) Single sind, (1) einen Partner haben oder (2) verheiratet sind oder in einer eingetragenen Lebensgemeinschaft leben. Alle Indikatoren hatten weniger als $1 \%$ fehlende Werte.

Beim akkumulierten Humankapital wurde zwischen dem Humankapital, das vor Eintritt in den Betrieb erworben wurde und dem, nach Eintritt in den Betrieb erworbenen Humankapital unterschieden. Für das Erstgenannte differenzieren wir zwischen niedriger, mittlerer und hoher Bildung auf Basis der Bildungsklassifikation CASMIN, die Angaben sowohl zur allgemeinen als auch zur beruflichen Ausbildung berücksichtigt: CASMIN 1 (Hauptschulabschluss und berufliche Ausbildung, Hauptschulabschluss ohne berufliche Ausbildung, kein Abschluss), CASMIN 2 (Fachhochschulreife/Abitur und berufliche Ausbildung, Mittlere Reife und berufliche Ausbildung, Mittlere Reife ohne berufliche Ausbildung) und CASMIN 3 (Fachhochschulabschluss, Hochschulabschluss). Die 22 Beschäftigten mit fehlenden Angaben zum Bildungsabschluss wurden nicht in der Analyse berücksichtigt. Arbeitserfahrung vor Eintritt in den Betrieb misst die Jahre der aktiven Arbeits-

${ }^{8}$ Für die LEEP-B3 Daten wurde $d a s$ Einkommen der Beschäftigten in der Beschäftigtenbefragung erhoben, um auch zwischen sehr hohen Einkommen differenzieren zu können und um Bonuszahlungen mit zu berücksichtigen. Die Registerdaten zum Einkommen des IAB sind in Abhängigkeit vom Spitzensteuersatz nach oben hin gekappt. 
Tab. 1 Deskriptive Informationen zu den Beschäftigten $(N=5099)$

\begin{tabular}{|c|c|c|c|c|}
\hline & $\begin{array}{l}\text { Frauen mit } \\
\text { Kinder } \\
(N=1542) \\
\text { Mittelwert } \\
\%\end{array}$ & $\begin{array}{l}\text { Männer mit } \\
\text { Kinder } \\
(N=1774) \\
\text { Mittelwert } \\
\%\end{array}$ & $\begin{array}{l}\text { Frauen ohne } \\
\text { Kinder } \\
(N=833) \\
\text { Mittelwert } \\
\%\end{array}$ & $\begin{array}{l}\text { Männer } \\
\text { ohne Kinder } \\
(N=950) \\
\text { Mittelwert } \\
\%\end{array}$ \\
\hline \multicolumn{5}{|l|}{ Einkommen } \\
\hline Stundenlohn & 20,606 & 29,686 & 20,398 & 23,907 \\
\hline Ln (Stundenlohn) & 2,979 & 3,302 & 2,968 & 3,061 \\
\hline \multicolumn{5}{|l|}{ Beziehungsstatus } \\
\hline Single & $12 \%$ & $5 \%$ & $31 \%$ & $35 \%$ \\
\hline $\begin{array}{l}\text { Verheiratet/eingetragene Lebenspart- } \\
\text { nerschaft }\end{array}$ & $72 \%$ & $85 \%$ & $26 \%$ & $21 \%$ \\
\hline Partner & $16 \%$ & $10 \%$ & $43 \%$ & $44 \%$ \\
\hline Alter des jüngsten Kindes & 13,44 & 11,05 & 1 & 1 \\
\hline \multicolumn{5}{|l|}{ Bildung } \\
\hline CASMIN 1: niedrig & $10 \%$ & $18 \%$ & $6 \%$ & $13 \%$ \\
\hline CASMIN 2: mittel & $59 \%$ & $44 \%$ & $54 \%$ & $52 \%$ \\
\hline CASMIN 3: Hoch & $32 \%$ & $38 \%$ & $40 \%$ & $35 \%$ \\
\hline \multicolumn{5}{|l|}{ Arbeitserfahrung in Jahren } \\
\hline Vor Eintritt in Betrieb & 8,385 & 10,415 & 6,337 & 6,503 \\
\hline Nach Eintritt in Betrieb (Tenure) & 8,730 & 10,002 & 5,811 & 5,893 \\
\hline Arbeitsstunden & 29,413 & 38,227 & 36,223 & 37,782 \\
\hline Überstunden & 3,873 & 5,314 & 5,055 & 4,683 \\
\hline \multicolumn{5}{|l|}{ Berufsmerkmale } \\
\hline Berufsstatus & 51,735 & 50,493 & 54,527 & 49,559 \\
\hline Führungsposition & $28 \%$ & $49 \%$ & $29 \%$ & $32 \%$ \\
\hline \multicolumn{5}{|l|}{ Beruf } \\
\hline $\begin{array}{l}\text { Angehörige der gesetzgebenden } \\
\text { Körperschaft, leitende Verwaltungs- } \\
\text { bedienstete und Führungskräfte }\end{array}$ & $1 \%$ & $5 \%$ & $0,36 \%$ & $2 \%$ \\
\hline Akademische Berufe/Wissenschaftler & $22 \%$ & $27 \%$ & $28 \%$ & $27 \%$ \\
\hline $\begin{array}{l}\text { Techniker und gleichrangige nicht- } \\
\text { technische Beruf }\end{array}$ & $39 \%$ & $24 \%$ & $35 \%$ & $21 \%$ \\
\hline Bürokräfte und verwandte Berufe & $19 \%$ & $10 \%$ & $20 \%$ & $13 \%$ \\
\hline Dienstleistungsberufe und Verkäufer & $8 \%$ & $5 \%$ & $11 \%$ & $6 \%$ \\
\hline $\begin{array}{l}\text { Fachkräfte in Land- und Forstwirt- } \\
\text { schaft und Fischerei }\end{array}$ & $0,32 \%$ & $0,51 \%$ & $0,24 \%$ & $0,21 \%$ \\
\hline Handwerks-und verwandte Berufe & $2 \%$ & $15 \%$ & $2 \%$ & $18 \%$ \\
\hline $\begin{array}{l}\text { Bediener von Anlagen und Maschi- } \\
\text { nen und Montageberufe }\end{array}$ & $2 \%$ & $9 \%$ & $1 \%$ & $8 \%$ \\
\hline Hilfsarbeitskräfte & $7 \%$ & $6 \%$ & $4 \%$ & $6 \%$ \\
\hline
\end{tabular}


marktpartizipation ohne Perioden von Arbeitslosigkeit oder Elternzeit und basiert auf den IAB Registerdaten. Das akkumulierte Humankapital nach Eintritt in den Betrieb wurde über die um Elternzeiten bereinigte Betriebszugehörigkeit gemessen. Diese Angaben beruhen ebenfalls auf den Registerdaten des IAB. Arbeitszeit misst die vertraglichen Arbeitsstunden pro Woche sowie den Umfang der Überstunden. Höhere Werte als $50 \mathrm{~h}$ pro Woche wurden auf $50 \mathrm{~h}$ gesetzt. Zudem wurde ein quadrierter Effekt von Arbeitsstunden in die Analyse aufgenommen, da Arbeitsstunden eine nicht lineare Beziehung zum Einkommen aufweisen. 22 Beschäftigte gaben keine Angaben zu Arbeitsstunden. Zudem wurden zwei Ausreißer mit inkonsistenten Informationen von der Analyse ausgeschlossen. Überstunden wurden als Differenz zwischen vertraglicher und tatsächlicher Arbeitszeit abgebildet. Höhere Werte als $30 \mathrm{~h}$ pro Woche wurden auf $30 \mathrm{~h}$ gesetzt.

An Berufsmerkmalen werden der Beruf, der Berufsstatus und das Innehaben einer Führungsposition berücksichtigt. Die Führungsposition wurde über die folgende Frage ermittelt: „Gehört zu Ihren momentanen Aufgaben auch die Führung von Mitarbeitern oder die Leitung eines Teams, eines Bereichs oder Betriebsteiles?“ ( $1=\mathrm{Ja} ; 0=$ Nein). Um typische Arbeitsaufgaben zu messen und dafür zu kontrollieren, dass bestimmte Tätigkeiten nicht in Form von Teleheimarbeit durchgeführt werden können, wie z. B. die Bedienung von Anlagen und Maschinen, wurde der einstellige ISCO 2008 auf Basis von Registerdaten genutzt. Diese Klassifikation unterscheidet folgende Berufsgruppen: (1) Angehörige einer gesetzgebenden Körperschaft, leitende Verwaltungsbedienstete und Führungskräfte, (2) Akademische Berufe/Wissenschaftler, (3) Techniker und gleichrangige nichttechnische Beruf, (4) Bürokräfte und verwandte Berufe, (5) Dienstleistungsberufe und Verkäufer, (6) Fachkräfte in Land- und Forstwirtschaft und Fischerei, (7) Handwerks-und verwandte Berufe, (8) Bediener von Anlagen und Maschinen und Montageberufe, (9) Hilfsarbeitskräfte. Eine verlässliche Information zum Beruf stand für 31 Beschäftigte nicht zur Verfügung. Für die Abbildung des Berufsstatus wurde der ISEI verwendet, der auf Basis des vierstelligen ISCO-Codes zugeordnet wurde. Die deskriptiven Informationen für die Beschäftigtenvariablen enthält Tab. 1 und zwar separat für Frauen und Männer mit und ohne Kinder.

Organisationsvariablen Das Ausmaß und die Umsetzung von Teleheimarbeit wurden mit Hilfe von sowohl Betriebserhebung als auch Beschäftigtenbefragung abgebildet. Im Rahmen der Betriebserhebung wurde Unternehmensvertretern (meistens Vertreter der Personalabteilung) folgende Frage gestellt: „Auf dieser Liste finden Sie verschiedene familienfreundliche Maßnahmen. Sagen Sie mir bitte, was es in Ihrem Betrieb/in Ihrer Dienststelle gibt.“ Unter anderem konnte Telearbeit/Heimarbeit als Maßnahme ausgewählt werden. Damit wurde Teleheimarbeit einerseits explizit in diesen Kontext familienfreundlicher Maßnahmen gestellt und somit von anderen Formen von Telearbeit abgegrenzt. Andererseits bleibt bei dieser Abfrage unklar, in welchem Zeitumfang Teleheimarbeit möglich ist, und es bleibt auch offen, ob es sich um formalisierte oder eher informelle Regelungen handelt. Wie aus Tab. $2 \mathrm{zu}$ entnehmen ist, stellen $64 \%$ der Betriebe dar, dass Teleheimarbeit Teil der familienfreundlichen Personalpolitik ist. Der Anteil der Beschäftigten, die angeben, dass es Teleheimarbeit im Betrieb gibt, liegt hingegen bei 40,2\%. Um den Unterschied in 
der Selbstdarstellung und der tatsächlichen Praxis im Betrieb abzubilden, wurde zum einen die Betriebsinformation zur Verfügbarkeit von Teleheimarbeit genutzt. Zum anderen wurde die Variable Implementationslücke gebildet. Dafür wurde zuerst der Anteil von Beschäftigten im Betrieb aggregiert, die die Frage ,gibt es in Ihrem Unternehmen Telearbeit/Heimarbeit" mit ,ja“ beantwortet haben. Demnach ist in fast allen Betrieben Teleheimarbeit Teil der betrieblichen Praxis, doch das Ausmaß der Praxis und die Formalisierung als Personalpraktik variiert (siehe Tab. 2 mit einer Übersicht zu Informationen auf Betriebsebene). Erwartungsgemäß ist der durchschnittliche Anteil der Beschäftigten, die von der Option Teleheimarbeit wissen, in denjenigen Betrieben größer, in denen Teleheimarbeit laut Angabe der befragten Unternehmensvertreter Teil der familienfreundlichen Personalpolitik ist. Im zweiten Schritt wurde die Betriebsangabe zur Verfügbarkeit mit den aggregierten Informationen aus der Beschäftigtenbefragung gewichtet und von 1 subtrahiert (1 - (AG * AN)). Dieser Wert bildet ab, inwiefern es eine Lücke zwischen Selbstdarstellung und Praxis gibt. Je höher dieser Wert ist, desto größer ist auch die Implementationslücke in dem Sinne, dass Teleheimarbeit zwar als Teil der familienfreundlichen Personalpolitik dargestellt wird, jedoch Beschäftigte davon keine Kenntnis haben. Diejenigen Betriebe, in denen Teleheimarbeit nicht Teil der familienfreundlichen Personalpolitik ist, wurden bei dieser Variable auf den Mittelwert gesetzt. Tabelle 2 dokumentiert, dass es diesbezüglich große Unterschiede zwischen den Betrieben gibt.

Die Nutzung von Teleheimarbeit auf Basis von Beschäftigteninformationen misst den Anteil von Beschäftigten im Betrieb, die Teleheimarbeit auch nutzen (Werte zwischen 0 und 1). Die durchschnittliche Nutzung von Teleheimarbeit im Betrieb liegt bei 14,4 \% der Beschäftigten. Damit liegt der Anteil etwas höher als der Anteil von Teleheimarbeitern, die auf Basis des European Working Conditions Survey (EWCS) für 2015 identifiziert wurden (ca. 8\%; Chung und Van der Lippe 2018). Die etwas höheren Zahlen lassen sich durch unseren Fokus auf Großbetriebe erklären, in denen generell flexible Arbeitsplatzarrangements häufiger zur Verfügung stehen als in Klein- und Mittelständischen Betrieben. Der D21 Digital Index 2018/19 für Deutschland, gefördert durch das Bundesministerium für Wirtschaft und Energie und durchgeführt durch TNS, weist für Deutschland auf dem Land $12 \%$ Telearbeit, Heimarbeit oder mobiles Arbeiten aus, während in der Stadt $20 \%$ diese Arbeitsformen nutzen (Initiative D21 e. V. 2019). Dass in den hier verwendeten LEEP-B3Daten ein höherer Anteil Männer Teleheimarbeit nutzt, ist nicht ungewöhnlich und bestätigt sich auch in Angaben des EWCS (Chung und Van der Lippe 2018), des D21 Digital Index (Initiative D21 e. V.2019), der Sustainable Workforce-Daten (Van der Lippe und Lippényi 2018) sowie in weiteren Studien. Nach diesen Studien bestätigt sich auch, dass der Zugang zu Teleheimarbeit eher Beschäftigten in hohen Berufspositionen gewährt wird (Chung und Van der Lippe 2018; Lott 2019). Minimale und maximale Werte machen jedoch deutlich, dass die Nutzung von Teleheimarbeit stark zwischen Betrieben variiert.

Für die Überprüfung der Hypothesen bezüglich der Variation in der Umsetzungen von Teleheimarbeit und den Implikationen für geschlechtsspezifische Einkommensungleichheiten wurden mehrere Organisationsvariablen generiert: (1) Umsetzung 
Tab. 2 Deskriptive Informationen auf Organisationsebene $(N=97)$

\begin{tabular}{|c|c|c|c|c|}
\hline & \multicolumn{2}{|c|}{ Mittelwert/\% S. D. } & Min & Max \\
\hline \multicolumn{5}{|l|}{ Verfügbarkeit von Teleheimarbeit im Betrieb } \\
\hline AG: Personalpolitik Heimarbeit - ja & $64 \%$ & - & - & - \\
\hline $\begin{array}{l}\text { AN: Teleheimarbeit vorhanden (Anteil Beschäftigter } \\
\text { die angeben, dass es Teleheimarbeit im Unternehmen } \\
\text { gibt) }\end{array}$ & 0,402 & 0,304 & 0,014 & 1 \\
\hline $\begin{array}{l}\text { Wenn Teil familienfreundlicher Personalpolitik } \\
(N=62)\end{array}$ & 0,538 & 0,296 & 0,09 & 1 \\
\hline $\begin{array}{l}\text { Wenn kein Teil familienfreundlicher Personalpolitik } \\
(N=35)\end{array}$ & 0,160 & 0,099 & 0,014 & 0,365 \\
\hline AG/AN: Implementationsgap $(1-(\mathrm{AG} * \mathrm{AN}))(N=62)$ & 0,462 & 0,296 & 0 & 0,909 \\
\hline \multicolumn{5}{|l|}{ Umfang der Nutzung von Teleheimarbeit im Betrieb } \\
\hline $\begin{array}{l}\text { AN: Nutzung Teleheimarbeit (Anteil Beschäftigter die } \\
\text { angibt Teleheimarbeit zu nutzen) }\end{array}$ & 0,144 & 0,201 & 0 & 1 \\
\hline $\begin{array}{l}\text { Wenn Teil familienfreundlicher Personalpolitik } \\
(N=62)\end{array}$ & 0,197 & 0,234 & 0 & 1 \\
\hline $\begin{array}{l}\text { Wenn kein Teil familienfreundlicher Personalpolitik } \\
(N=35)\end{array}$ & 0,051 & 0,052 & 0 & 0,224 \\
\hline Anteil Frauen mit Kinder die Teleheimarbeit nutzt & 0,132 & 0,216 & 0 & 1 \\
\hline Anteil Männer mit Kinder die Teleheimarbeit nutzt & 0,162 & 0,226 & 0 & 1 \\
\hline Anteil Frauen ohne Kinder die Teleheimarbeit nutzt & 0,111 & 0,204 & 0 & 1 \\
\hline Anteil Männer ohne Kinder die Teleheimarbeit nutzt & 0,138 & 0,219 & 0 & 1 \\
\hline \multicolumn{5}{|c|}{ Umsetzung von Teleheimarbeit im Betrieb ( $N=82$ Betriebe in denen Teleheimarbeit genutzt wird) } \\
\hline $\begin{array}{l}\text { Umsetzung als Unterstützung (Anteil Nutzer die Vor- } \\
\text { gesetztenunterstützung für die Vereinbarkeit von Beruf } \\
\text { und Privatem angibt) }\end{array}$ & 0,722 & 0,240 & 0 & 1 \\
\hline $\begin{array}{l}\text { Umsetzung als Stigmatisierung (Anteil Nutzer die } \\
\text { angeben, dass man im Betrieb als weniger engagiert } \\
\text { angesehen wird, wenn man flexible Arbeitsplatzarran- } \\
\text { gements nutzt) }\end{array}$ & 0,135 & 0,190 & 0 & 1 \\
\hline $\begin{array}{l}\text { Umsetzung als Entgrenzung (Anteil Nutzer die ange- } \\
\text { ben, dass im Betrieb bei ähnlicher beruflichen Position } \\
\text { hohe Erwartungen an Mehrarbeit gestellt werden) }\end{array}$ & 0,791 & 0,204 & 0 & 1 \\
\hline
\end{tabular}

$A G$ Arbeitgeberinformation, $A N$ Arbeitnehmerinformation, $A G / A N$ Abgleich Information Arbeitgeber vs. Arbeitnehmer

als Unterstützung als Anteil der Nutzer von Teleheimarbeit im Betrieb, die Vorgesetztenunterstützung für die Vereinbarkeit von Beruf und Privatem angeben. Dieser Indikator lehnt sich an gängige Messinstrumente familienfreundlicher Organisationskulturen an (siehe z. B. Kossek et al. 2010; Van der Lippe und Lippényi 2018);

(2) Umsetzung als Stigmatisierung anhand des Anteils der Nutzer, die angeben, dass man im Betrieb als weniger engagiert angesehen wird, wenn man flexible Arbeitsplatzarrangements wie Teleheimarbeit nutzt. Diese Operationalisierung steht in Einklang mit Messinstrumenten des „Flexibilitäts-Stigmas“ (Chung und Van der Horst 2018); und (3) Umsetzung als Entgrenzung definiert als Anteil der Nutzer, die angeben, dass im Betrieb bei ähnlicher beruflicher Position hohe Erwartungen an Mehrarbeit gestellt werden. Dieses Messinstrument lehnt sich an Untersuchungen des „Flexibilitäts-Paradoxes“ an (siehe Chung und Van der Horst 2018). 


\subsection{Analysemethode}

Zur Überprüfung der Hypothesen verwenden wir hierarchische lineare Regressionen mit organisationsbezogenen fixed-effects und robusten Standardfehlern. Damit werden nur Einkommensungleichheiten nach Geschlecht-Familien-Kombination innerhalb von Betrieben und nicht zwischen Betrieben untersucht, da letztere für unsere Forschungsfrage nicht relevant sind. Weitere Vorteile dieser Methode sind, dass sie für unbeobachtete Heterogenität auf Betriebsebene kontrollieren sowie dafür, dass Männer und Frauen sich unterschiedliche Betriebe aussuchen (Selbstselektion; Allison 2005; Castilla 2008; England et al. 1988).

Tabelle 3 stellt Einkommensunterschiede zwischen Männern und Frauen mit und ohne Kinder innerhalb von Arbeitsorganisationen unter Berücksichtigung von Unterschieden in der familiären und beruflichen Situation sowie im akkumulierten Humankapital dar. Tabelle 4 beinhaltet die Ergebnisse zur Relevanz der Umsetzung von Teleheimarbeit für Einkommensungleichheiten zwischen Männern und Frauen mit und ohne Kinder. Mit Hilfe von Interaktionseffekten zwischen der GeschlechtFamilien-Kombinationsvariable und den jeweiligen Organisationsvariablen werden die oben entwickelten Hypothesen getestet. Die Interaktion zwischen GeschlechtFamilien-Kombination und Teleheimarbeit als Teil familienfreundlicher Personalpolitik kontrolliert dafür, dass in Betrieben ohne formale Regelungen die Nutzung generell niedriger ausfällt und so ein mittlerer Anteil in der Nutzung für Betriebe ohne formale Regelungen bereits als hoch einzuschätzen ist. Der direkte Effekt der Geschlecht-Familien-Kombination stellt daher Einkommensungleichheiten für Betriebe ohne formale Regelungen in der familienfreundlichen Personalpolitik dar. Aus den deskriptiven Informationen ist jedoch bekannt, dass in diesen Betrieben Teleheimarbeit durchaus informell praktiziert werden kann. Des Weiteren ist darauf hinzuweisen, dass der Haupteffekt der Organisationsvariablen nicht enthalten ist, da Organisations-fixed-effects nicht den Effekt von Organisations-Kovariaten berechnen ${ }^{9}$.

\section{Ergebnisse}

Die Ergebnisse in Tab. 3 zeigen, dass Männer mit Kindern im Vergleich zu Frauen mit Kindern einen 15,1 \% höheren Stundenlohn beziehen. Bei Männern ohne Kinder ist der Stundenlohn um 6,9 \% höher als bei Frauen mit Kindern. Der Stundenlohn zwischen Frauen ohne und mit Kindern ist nicht signifikant unterschiedlich. Dies gilt allerdings nur für den Fall, dass bereits Unterschiede in beruflichen Merkmalen, wie Arbeitsstunden und ausgeübter Beruf, mit berücksichtigt wurden.

Tabelle 4 enthält zusätzlich die Interaktionen der Geschlecht-Familien-Kombination mit den Organisationsvariablen, die die Umsetzung von Teleheimarbeit und ihre Implikationen für geschlechtsspezifische Einkommensungleichheiten abbilden. Die Interaktion zwischen der Geschlecht-Familien-Kombination und der betriebsseitig dargestellten Verfügbarkeit von Teleheimarbeit in der Betriebsbefragung gibt an,

${ }^{9} \mathrm{Zu}$ ähnlichem Vorgehen siehe Abendroth et al. (2017). 
Tab. 3 Einkommensungleichheiten zwischen Männern und Frauen mit und ohne Kinder - Fixed effectRegressionen

\begin{tabular}{|c|c|c|}
\hline Variable & $\mathrm{B}$ & (SE) \\
\hline \multicolumn{3}{|l|}{ Geschlecht-Familien-Kombination (Ref. K. Frau mit Kind) } \\
\hline Mann mit Kind & $0,151 * * *$ & $(0,017)$ \\
\hline Frau ohne Kind & 0,008 & $(0,015)$ \\
\hline Mann ohne Kind & $0,069^{* *}$ & $(0,021)$ \\
\hline Alter des jüngsten Kindes & $-0,001$ & $(0,001)$ \\
\hline \multicolumn{3}{|l|}{ Beziehungsstatus (Ref. C. Single) } \\
\hline Verheiratet/eingetragene Lebenspartnerschaft & $0,030^{*}$ & $(0,013)$ \\
\hline Partner & $0,045^{* *}$ & $(0,013)$ \\
\hline \multicolumn{3}{|l|}{ Bildung (Ref. K. niedrig/Casmin 1) } \\
\hline Mittel/Casmin 2 & $0,095 * * *$ & $(0,016)$ \\
\hline Hoch/Casmin 3 & $0,286 * * *$ & $(0,022)$ \\
\hline \multicolumn{3}{|l|}{ Arbeitserfahrung in Jahren } \\
\hline Vor Eintritt in Betrieb & $0,008 * * *$ & $(0,001)$ \\
\hline Nach Eintritt in Betrieb (Tenure) & $0,012 * * *$ & $(0,001)$ \\
\hline Arbeitsstunden & $-0,029 * *$ & $(0,009)$ \\
\hline Arbeitsstunden $^{2}$ & $0,000 * *$ & $(0,000)$ \\
\hline Überstunden & $0,014 * * *$ & $(0,001)$ \\
\hline \multicolumn{3}{|l|}{ Berufsmerkmale } \\
\hline Berufsstatus & $0,007 * * *$ & $(0,002)$ \\
\hline Führungsposition & $0,100 * * *$ & $(0,011)$ \\
\hline \multicolumn{3}{|l|}{ Beruf (Ref. K. Hilfsarbeitskräfte) } \\
\hline $\begin{array}{l}\text { Angehörige der gesetzgebenden Körperschaft, leitende Verwal- } \\
\text { tungsbedienstete und Führungskräfte }\end{array}$ & 0,109 & $(0,097)$ \\
\hline Akademische Berufe/Wissenschaftler & $-0,110$ & $(0,092)$ \\
\hline Techniker und gleichrangige nichttechnische Beruf & $-0,067$ & $(0,064)$ \\
\hline Bürokräfte und verwandte Berufe & $-0,037$ & $(0,049)$ \\
\hline Dienstleistungsberufe und Verkäufer & $-0,019$ & $(0,035)$ \\
\hline Fachkräfte in Land- und Forstwirtschaft und Fischerei & 0,012 & $(0,044)$ \\
\hline Handwerks-und verwandte Berufe & $-0,028$ & $(0,038)$ \\
\hline Bediener von Anlagen und Maschinen und Montageberufe & $-0,017$ & $(0,030)$ \\
\hline Konstante & $2,300 * * *$ & $(0,200)$ \\
\hline$n$ (Personen) & 5099 & - \\
\hline$N$ (Organisationen) & 97 & - \\
\hline Rho & 0,284 & - \\
\hline$r 2{ }^{2} w$ & 0,334 & - \\
\hline$r 2 \_o$ & 0,384 & - \\
\hline$r 2 \_b$ & 0,552 & - \\
\hline
\end{tabular}

$p<0,10, * p<0,05,{ }^{* *} p<0,01, * * * p<0,001$ 
Tab. 4 Interaktionseffekte (alle in einem Modell) ${ }^{\mathrm{a}}$

\begin{tabular}{|c|c|c|}
\hline Variable & B & $(\mathrm{SE})$ \\
\hline \multicolumn{3}{|c|}{ Geschlecht-Familien-Kombination (Ref. K. Frau mit Kind) } \\
\hline Mann mit Kind & $0,137 * * *$ & 0,026 \\
\hline Frau ohne Kind & 0,005 & 0,023 \\
\hline Mann ohne Kind & 0,034 & 0,034 \\
\hline \multicolumn{3}{|c|}{ Interaktionen Teleheimarbeit } \\
\hline \multicolumn{3}{|c|}{ Dargestellte Verfügbarkeit von Teleheimarbeit im Betrieb mit } \\
\hline Mann mit Kind & 0,028 & 0,031 \\
\hline Frau ohne Kind & 0,006 & 0,027 \\
\hline Mann ohne Kind & 0,066 & 0,039 \\
\hline \multicolumn{3}{|c|}{ Implementationslücke Teleheimarbeit mit } \\
\hline Mann mit Kind & 0,087 & 0,078 \\
\hline Frau ohne Kind & $-0,010$ & 0,074 \\
\hline Mann ohne Kind & $-0,083$ & 0,085 \\
\hline \multicolumn{3}{|c|}{ Umsetzung von Teleheimarbeit als Stigmatisierung mit } \\
\hline Mann mit Kind & 0,043 & 0,081 \\
\hline Frau ohne Kind & $-0,036$ & 0,040 \\
\hline Mann ohne Kind & $-0,015$ & 0,116 \\
\hline \multicolumn{3}{|c|}{ Umsetzung von Teleheimarbeit als Entgrenzung mit } \\
\hline Mann mit Kind & $0,070+$ & 0,053 \\
\hline Frau ohne Kind & $-0,000$ & 0,042 \\
\hline Mann ohne Kind & $0,121^{*}$ & 0,054 \\
\hline \multicolumn{3}{|c|}{ Umsetzung von Teleheimarbeit als Unterstützung } \\
\hline Mann mit Kind & $-0,106^{*}$ & 0,055 \\
\hline Frau ohne Kind & $-0,047+$ & 0,031 \\
\hline Mann ohne Kind & $-0,073$ & 0,061 \\
\hline \multicolumn{3}{|c|}{ Anteil Beschäftigter die Teleheimarbeit nutzt mit } \\
\hline Mann mit Kind & 0,019 & 0,090 \\
\hline Frau ohne Kind & $-0,042$ & 0,110 \\
\hline Mann ohne Kind & $-0,271^{*}$ & 0,141 \\
\hline$n$ (Personen) & 5099 & - \\
\hline$N$ (Organisationen) & 97 & - \\
\hline Rho & 0,289 & - \\
\hline$r 2 \_w$ & 0,336 & - \\
\hline$r 2 \_o$ & 0,547 & - \\
\hline$r 2 \_b$ & 0,381 & - \\
\hline
\end{tabular}

${ }^{a}$ Kontinuierliche Variablen zentriert; einseitiger Signifikanztest für gerichtete Hypothesen; Kontrollvariablen aus Tab. 3 enthalten

$+p<0,10, * p<0,05, * * p<0,01$ 


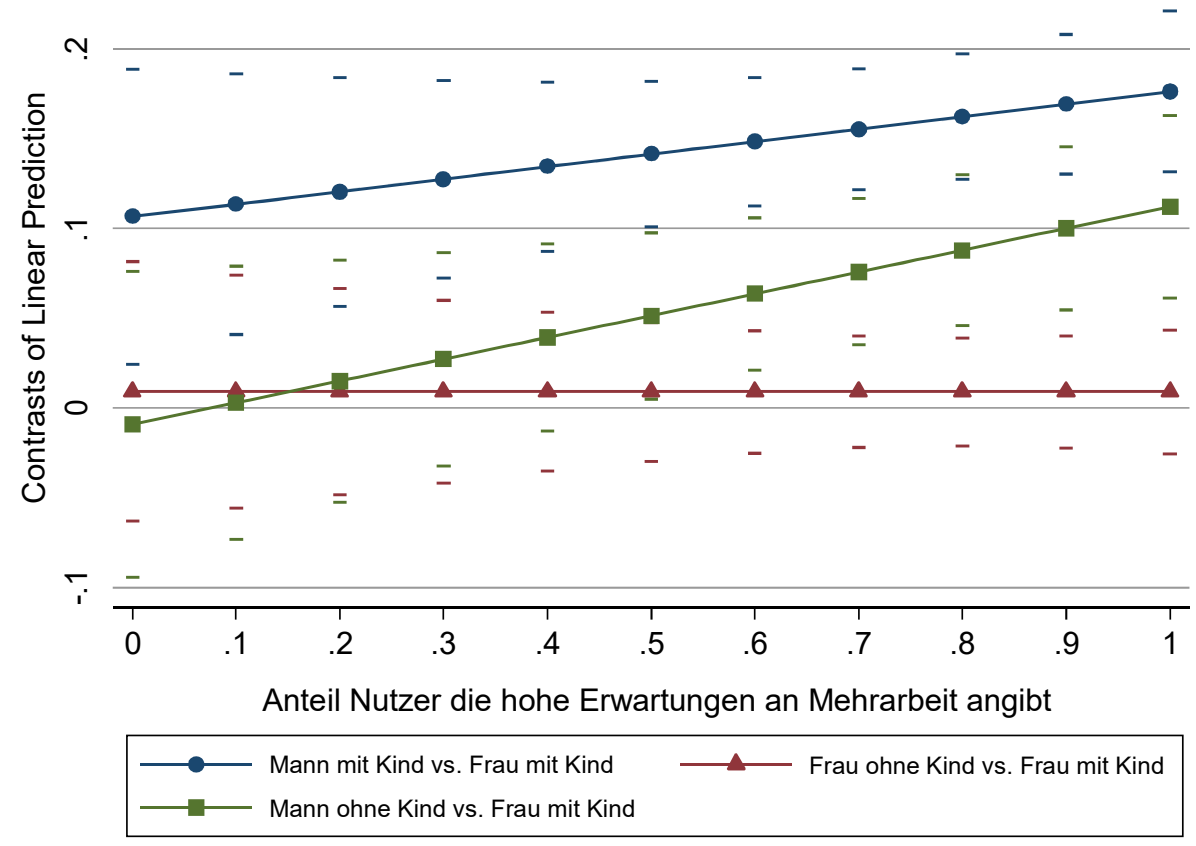

Abb. 1 Bereinigte Einkommensungleichheiten zwischen den Geschlechtern in Abhängigkeit von einer Umsetzung als Entgrenzung von Teleheimarbeit (Fixed effect-Regressionen mit 95\% Konfidenzintervallen)

inwiefern Einkommensungleichheiten zwischen den Geschlechtern zwischen Betrieben variieren, in denen Teleheimarbeit Teil betrieblicher Personalpolitik oder nur Teil informeller betrieblicher Praxis ist. Die deskriptiven Darstellungen haben gezeigt, dass in allen Betrieben Teleheimarbeit genutzt wird, der Umfang der Nutzung in Betrieben jedoch höher ist, wenn Teleheimarbeit Teil der offiziellen betrieblichen Personalpolitik ist. Die Ergebnisse in Tab. 4 zeigen jedoch keinen signifikanten Einfluss dieser Unterscheidung auf Einkommensungleichheiten zwischen den Geschlechtern. Das Gleiche gilt für das Vorhandensein einer Implementationslücke. Hypothese $H$ 1, nach der Einkommensbenachteiligungen von Frauen im Vergleich zu Männern in Betrieben größer sind, in denen die Selbstdarstellung zur Verfügbarkeit von Teleheimarbeit nicht mit einer tatsächlichen Verfügbarkeit im Betrieb einhergeht, wird somit nicht bestätig ${ }^{10}$. Auch eine Umsetzung von Teleheimarbeit, die mit einer Stereotypisierung als weniger beruflich engagiert einhergeht, hat keinen Einfluss auf geschlechtsspezifische innerbetriebliche Einkommensungleichhei-

\footnotetext{
${ }^{10}$ Die Interaktion zwischen Geschlecht-Familien-Kombination und Implementationslücke ist schwach signifikant $(p<0,1)$, wenn Angaben zur Nutzung von Telearbeit im Betrieb nicht berücksichtigt werden. Dies lässt sich durch die hohe Korrelation der beiden Angaben erklären. Die Ergebnisse legen nahe, dass der Umfang der Nutzung von Teleheimarbeit für geschlechtsspezifische Einkommensungleichheiten entscheidender ist als der Umfang, in dem die Verfügbarkeit von Teleheimarbeit im Betrieb bekannt ist.
} 


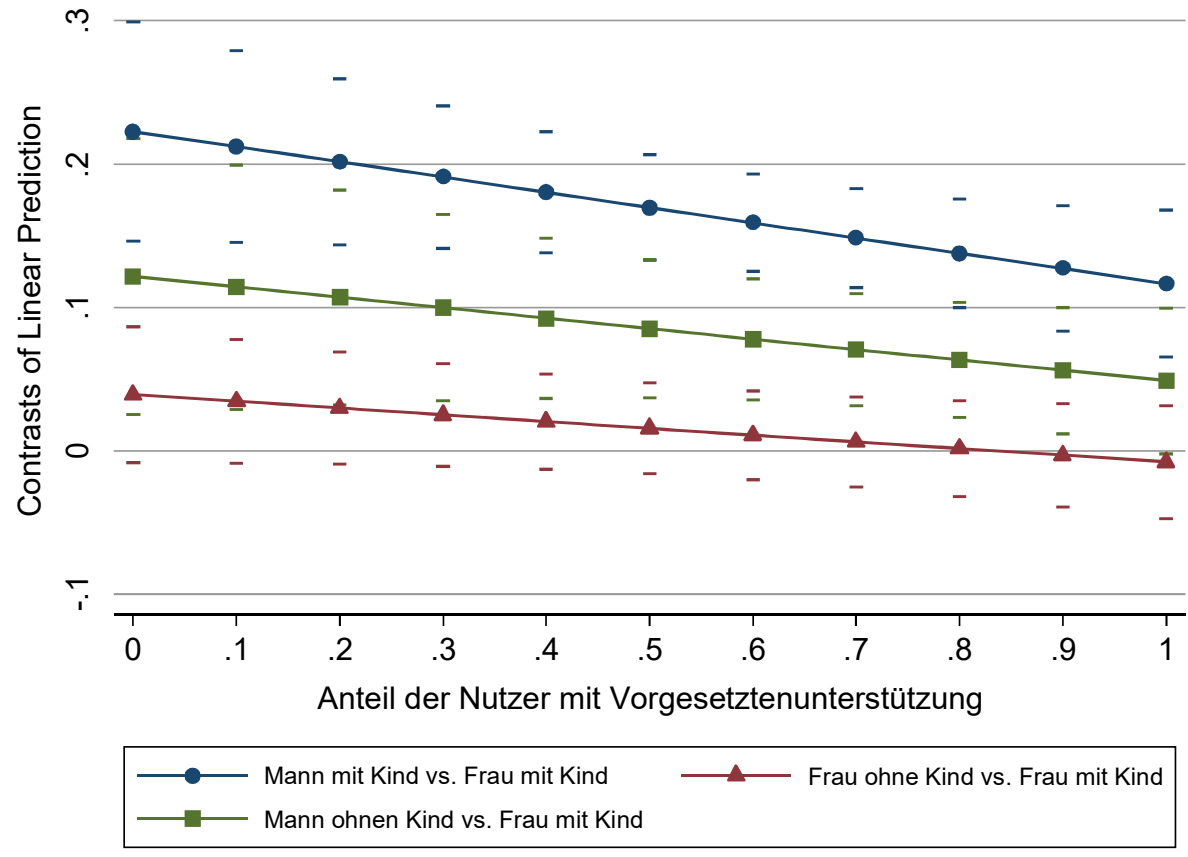

Abb. 2 Bereinigte Einkommensungleichheiten zwischen den Geschlechtern in Abhängigkeit einer Umsetzung als Unterstützung von Teleheimarbeit (Fixed effect-Regressionen mit 95\% Konfidenzintervallen)

ten und zwar unabhängig vom Elternstatus. Somit muss auch die StigmatisierungsHypothese (H 2) verworfen werden.

Eine Umsetzung von Teleheimarbeit als Entgrenzung moderiert jedoch geschlechtsspezifische Einkommensungleichheiten. Abbildung 1 zeigt die signifikante Interaktion zwischen Geschlecht-Familien-Kombination und entgrenzungsfördernder Umsetzung von Teleheimarbeit. In Betrieben, in denen eine Umsetzung von Teleheimarbeit mit hohen Erwartungen an Mehrarbeit verbunden ist, sind Einkommensungleichheiten zwischen Männern mit Kindern und Frauen mit Kindern größer. Dieser Befund ist jedoch mit $p<0,1$ nur schwach signifikant. Einkommensungleichheiten zwischen Männern mit Kind vs. Frauen mit Kind liegen bei 8,8 \%, wenn Teleheimarbeit nicht mit hohen Erwartungen an Mehrarbeit im Betrieb einhergeht ${ }^{11}$, und bei $15,8 \%$, wenn die Beschäftigten, die Teleheimarbeit im Betrieb nutzen, hohe Erwartungen an Mehrarbeit im Betrieb angeben. Das Gleiche gilt für Einkommensungleichheiten zwischen Männern ohne Kinder im Vergleich zu Frauen mit Kindern. Diese liegen bei 5,3\%, wenn Teleheimarbeit nicht mit hohen Erwartungen an Mehrarbeit im Betrieb einhergeht, und bei 17,4\%, wenn Beschäftigte mit Teleheimarbeit hohe Erwartungen an Mehrarbeit im Betrieb angeben. Dies bestätigt die Entgrenzungshypothese (H 3), nach der in Betrieben, in denen die Nutzung von Teleheimarbeit mit einer hohen Erwartung an Mehrarbeit einhergeht, Einkommensbenachteiligungen von Frauen im Vergleich zu Männern

${ }^{11}$ Berechnungsbeispiel: $0,137+0,070 \times-0,694=0,088$. 
größer sind. Die Interaktion mit dem Anteil der Beschäftigten, die Teleheimarbeit nutzen, kontrolliert dafür, dass der Nutzungsanteil von Teleheimarbeit im Betrieb in Abhängigkeit von der Umsetzung von Teleheimarbeit und dem Zeitraum, seit dem es Teleheimarbeit im Betrieb gibt, variieren kann. Die Ergebnisse zeigen insgesamt, dass unabhängig von Umsetzungsmustern in Betrieben, in denen ein hoher Teil der Beschäftigten Teleheimarbeit nutzt, Einkommensvorsprünge von Männern nicht mehr existent sind oder sich sogar umkehren.

Eine Umsetzung von Teleheimarbeit als Unterstützung moderiert ebenfalls geschlechtsspezifische Einkommensungleichheiten. Abbildung 2 visualisiert den in Tab. 4 dargestellten signifikanten Interaktionseffekt zwischen Geschlecht-FamilienKombination und einer Umsetzung von Teleheimarbeit als Unterstützung. Je größer der Anteil der Nutzer, die von ihren Vorgesetzten unterstützt werden, desto geringer sind Einkommensungleichheiten zwischen Männern und Frauen mit Kindern. Vergleicht man Einkommensbenachteiligungen von Frauen mit Kindern im Vergleich zu Männern mit Kindern in Abhängigkeit von der Unterstützung durch Vorgesetzte, zeigt sich folgendes Bild: In Betrieben, in denen keiner der Nutzer von Teleheimarbeit angibt, von Vorgesetzten in den Vereinbarkeitsbestrebungen unterstützt zu werden, ist die Differenz in der Einkommensbenachteiligung um 10,6 \% größer als in Betrieben, in denen alle Nutzer eine solche Unterstützung angeben. ${ }^{12}$

Zudem scheinen in Betrieben, in denen die Nutzung von Teleheimarbeit nicht mit einer Unterstützung für die Vereinbarkeit von Beruf und Privatem durch Vorgesetzte einhergeht, auch Einkommen zwischen Frauen mit und ohne Kinder ungleicher zu sein. Diese Ergebnisse stehen in Einklang mit der Unterstützungshypothese (H 4). Bemerkenswerterweise ist die Interaktion zwischen Geschlecht-Familien-Kombination und vereinbarkeitsunterstützender Umsetzung nur dann signifikant, wenn gleichzeitig eine Umsetzung als Entgrenzung - d. h. es gibt im Betrieb hohe Erwartungen an Mehrarbeit - mit im Modell berücksichtigt wird. Das bedeutet, nur wenn eine vereinbarkeitsunterstützende Umsetzung nicht zusätzlich mit einer Entgrenzungskultur einhergeht, verringert diese geschlechtsspezifische Einkommensungleichheiten.

\section{Diskussion}

Teleheimarbeit ist in Großbetrieben eine weit verbreitete personalpolitische Maßnahme zur Vereinbarkeit von Beruf und Privatem. Vor dem Hintergrund neuer Entwicklungen in digitalen Technologien wird sie weiter an Bedeutung gewinnen, weil sie für immer mehr Beschäftigte umsetzbar wird (Den Dulk et al. 2012; Kleemann 2005; Kossek 2016). Zudem wird sie zunehmend politisch forciert, was das Risiko einer heuchlerischen Umsetzung erhöht. Umso dringlicher stellt sich die Frage nach möglichen Konsequenzen für die Beschäftigten und nach der Rolle, die die unterschiedlichen betrieblichen Bedingungen spielen. In unserem Beitrag haben wir

\footnotetext{
$\overline{12}$ Das Einsetzen von Minimum und Maximum der genutzten zentrierten Variablen in der Gleichung von Tab. 4 ergibt rechnerisch auf Basis eines Minimums von $=-0,641$ und eines Maximums von 0,359: Betriebe, in denen keiner der Nutzer von Teleheimarbeit Unterstützung angibt: $0,137+-0,106 \times-0,641=0,205$ vs. Betriebe, in denen alle Nutzer Unterstützung angeben: $0,137+-0,106 \times 0,359=0,099$.
} 
uns insbesondere der Frage gewidmet, ob die Verfügbarkeit von Teleheimarbeit als flexibles Arbeitsplatzarrangement im Betrieb dazu beitragen kann, geschlechtsspezifische Einkommensungleichheiten abzubauen oder sie umgekehrt sogar akzentuiert. Bisherige Forschungsergebnisse dazu sind nicht eindeutig. Dies kann auch auf unterschiedliche Untersuchungsdesigns zurückzuführen sein, die nicht oder nur ungenügend für unbeobachtete Heterogenität kontrollieren. Wir haben hierzu das Design einer Arbeitgeber-Arbeitnehmer-Befragung genutzt und die Perspektive auf innerbetriebliche Ungleichheiten in Abhängigkeit von spezifischen, aber ansonsten kontrollierten betrieblichen Bedingungen gelegt. Gerade in dieser Hinsicht liefern die vorgelegten Analysen einen wesentlichen Beitrag zur bestehenden Forschung.

Ausgehend von einer organisationalen Ungleichheitsperspektive haben wir argumentiert, dass die Implikationen der Verfügbarkeit von Teleheimarbeit für bereinigte Einkommensungleichheiten zwischen Männern und Frauen zwischen Betrieben variieren, da Betriebe sich in ihrer Historie, ihrer Zusammensetzung, Kultur, ihren Umweltbeziehungen und somit auch in ihren Ungleichheitsregimen unterscheiden. In diesem Zusammenhang zeigt sich, dass Teleheimarbeit sehr unterschiedlich konnotiert sein kann, sowohl im Hinblick auf die Stereotypisierung von Beschäftigtengruppen als auch ihre Möglichkeiten, Zugänge zu Ressourcen auf Basis ihrer Gruppenzugehörigkeit zu monopolisieren, hier konkret: die Realisierung vergleichsweise höherer Einkommen. Dazu gehört auch, dass die Implementation von Teleheimarbeit eher „talk“ als ,action“ im Sinne des Neonstitutionalismus sein kann, d. h. nach außen demonstrativ als betriebliche Leistung hergezeigt, aber nach innen gegenüber der Belegschaft eher als nachrangige, weniger gute Beschäftigungsform kommuniziert wird. Eine Umsetzung von Telearbeit als Teil der betrieblichen Beschäftigungspolitik kann deshalb die relationalen Einkommensungleichheiten in Betrieben in verschiedene Richtungen beeinflussen.

Die von uns unterschiedenen vier Umsetzungsmuster von Teleheimarbeit: (a) dargestellte Verfügbarkeit mit Implementationslücke, (b) Umsetzung als Stigmatisierung, (c) Umsetzung als Entgrenzung sowie (d) Umsetzung als Unterstützung haben versucht, diese unterschiedlichen Perspektiven einer Umsetzung von Teleheimarbeit abzubilden, verbunden mit der Annahme, dass sie Männer und Frauen, Mütter und Väter in potenziell unterschiedlicher Weise begünstigen. Allerdings haben dies unsere Analysen nicht für alle Muster gleichermaßen bestätigt. Die Ergebnisse legen nahe, dass nur bei einer Umsetzung von Teleheimarbeit als Unterstützung geschlechtsspezifische Einkommensungleichheiten abgebaut werden. Nicht in jedem Betrieb dominiert die klassische Norm des „,idealen Beschäftigten“, der nur geringe familiäre Verpflichtungen hat. In manchen betrieblichen Kontexten wird mit Unterstützung des Managements in Familienfreundlichkeit investiert, um Mitarbeiter beschäftigungsfähig zu halten und/oder um ein attraktiver Arbeitgeber zu sein. Chancenhortung von Männern durch die Entsprechung einer klassischen Norm des sich konkurrenzlos dem Betrieb widmenden, immer anwesenden ,idealen Beschäftigten“ ist damit offensichtlich die Durchschlagskraft genommen, eine Einschätzung oder Stigmatisierung von Teleheimarbeit als weniger produktiv und weniger engagiert nicht mehr vorhanden zu sein. Ergebnisse aus anderen Untersuchungen haben gezeigt, dass solche auf Signale beruhenden Auf- oder Abwertungen insbesondere dann an Relevanz verlieren, wenn es stattdessen unvoreingenommenere, transpa- 
rentere Verfahren zur Leistungsbeurteilung gibt (Abendroth et al. 2017; Dobbin et al. 2015). Allerdings stellen sich die positiven Auswirkungen einer Umsetzung von Teleheimarbeit als Unterstützung nur dann ein, wenn nicht gleichzeitig eine Umsetzung als Entgrenzung in den jeweiligen Betrieben dominiert. Dann nämlich scheint die Umsetzung als Unterstützung nur zu bedeuten, dass eine Unterstützung basaler familiärer Verpflichtungen im Betrieb umgesetzt wird, um hohen Arbeitsanforderungen gerecht zu werden. Dies entspricht somit eher einer Zuspitzung der Norm konkurrenzloser Verpflichtung, dann aber in Verbindung mit Erreichbarkeit statt Anwesenheit.

Wird Teleheimarbeit als Entgrenzung mit hohen Erwartungen an Mehrarbeit im Betrieb umgesetzt, werden geschlechtsspezifische Einkommensungleichheiten sogar befördert. Hier ist davon auszugehen, dass gerade Beschäftigte mit hohen familiären Verpflichtungen, insbesondere Frauen mit Kindern, diese Ansprüche nicht erfüllen können und damit auch geringere Forderungen bei Einkommensverhandlungen stellen. Es sind dann eher Männer, die die Möglichkeit erhalten, hohes Arbeitsengagement im Sinne einer zugespitzten Norm des ,idealen Beschäftigten“ zu signalisieren und damit Einkommenschancen zu erhöhen, allerdings auf Kosten der Möglichkeit, sich in der Familie stärker einzubringen. Dies legen auch die Ergebnisse von Lott und Chung (2016) nahe, die zeigen, dass mit der Nutzung flexibler Arbeitsplatzarrangements für Männer positive Einkommenseffekte und für Frauen negative Einkommenseffekte verbunden sind, denn Männer nutzen im Vergleich zu Frauen häufiger und in größerem Umfang Teleheimarbeit als Arbeitsintensivierung (Lott 2019).

Im Einklang mit dem Neo-Institutionalismus und dem Stand der Forschung haben wir auch Betriebe identifiziert, in denen wir eine Implementationslücke zwischen offizieller und von den Beschäftigten wahrgenommener Verfügbarkeit von Teleheimarbeit finden (z. B. Den Dulk und De Ruiter 2008; McDonald et al. 2005). Wir finden jedoch keine Belege dafür, dass in diesen Betrieben Einkommensungleichheiten zwischen den Geschlechtern größer sind. Der Umfang der Nutzung scheint entscheidender zu sein als die Kenntnis über die Verfügbarkeit im Betrieb. In ähnlicher Weise findet wir in Betrieben zwar auch eine Umsetzung als Stigmatisierung (z. B. Blair-Loy und Wharton 2002; Konrad und Yang 2012; Pleck 1993), jedoch keine Auswirkungen auf geschlechtsspezifische Einkommensungleichheiten. Dies lässt sich möglicherweise dadurch erklären, dass die Umsetzung als Stigmatisierung in viel geringerem Umfang als die Umsetzung als Unterstützung und Entgrenzung stattfindet und Teleheimarbeit in dem Fall generell wenig genutzt wird.

Durch die Differenzierung zwischen Männern und Frauen mit und ohne Kinder wurde zudem deutlich, dass sich das Einkommen von Frauen mit Kindern nicht signifikant vom Einkommen von Frauen ohne Kinder unterscheidet, wenn um Humankapital und berufliche Merkmale bereinigtes Einkommen betrachtet wird. Somit konnte die Relevanz von Teleheimarbeit für Einkommensungleichheiten nur generell für Frauen nachgewiesen werden. Ein darüber hinausgehender Einkommens-Malus für Mütter im Zusammenhang mit Teleheimarbeit ist jedoch nicht sichtbar. Hier bedarf es zukünftige Forschung, die in den Blick nimmt, ob bereits bei Entscheidungen für Teilzeit oder bestimmte Berufe die Umsetzung von Teleheimarbeit im Betrieb eine Rolle spielt, um hier weitergehende Aussagen treffen zu können. Für Männer 
zeigen unsere Ergebnisse, dass mit der Elternschaft eher noch höhere Einkommensvorteile verbunden sind. Dies steht im Einklang mit anderen Forschungsergebnissen zu sogenannten ,fatherhood premium“-Effekten zwischen und innerhalb von Betrieben (z. B. Petersen et al. 2011). Die Verfügbarkeit von Teleheimarbeit wirkt sich jedoch größtenteils ähnlich für das Einkommen von Männern mit und ohne Kinder aus. Teleheimarbeit, die mit einer höheren Erwartung an Mehrarbeit verbunden ist, scheint allerdings besonders Männern ohne Kinder die Möglichkeit zu geben, glaubhaft eine hohe Arbeitsbereitschaft zu signalisieren, was mit Einkommensvorteilen belohnt wird.

Es lässt sich also übergreifend festhalten, dass die Verfügbarkeit von Teleheimarbeit dann geschlechtsspezifische Einkommensungleichheiten in Betrieben beeinflusst, wenn sie die Norm des idealen Beschäftigten noch weiter zuspitzt (hohe Erwartungen an Mehrarbeit) oder mit einer alternativen Wahrnehmung und Bewertung von Beschäftigten mit familiären Verpflichtungen einhergeht, die nicht der verbreiteten „Norm des idealen Beschäftigten“ entspricht.

Die verschiedenen beschriebenen Umsetzungsmuster stellen Idealtypen dar, die sich nicht ausschließen müssen, sondern auch gleichzeitig in Betrieben präsent sein können, z. B. unterschiedlich für Männer und Frauen (siehe auch Lott 2019). Sie können sich auch gegenseitig konterkarieren, wie wir am Beispiel des Zusammenspiels der Umsetzung als Unterstützung und als Entgrenzung gezeigt haben. In zukünftiger Forschung muss mit Längsschnittdaten weiter geprüft werden, inwiefern mit der Implementation und zunehmender Nutzung von Teleheimarbeit für Frauen und Männer bessere oder schlechtere Einkommenschancen einhergehen, und ob verschiedene Umsetzungsmuster für Männer und Frauen unterschiedlich relevant sind. Wünschenswert wären zudem präzisere, direktere Messungen der Umsetzungs-Modi von Teleheimarbeit und der dahinter vermuteten Mechanismen, insbesondere auf der Betriebsebene. Als Einschränkung der präsentierten Studie ist zudem anzumerken, dass keine Angaben zum Zeitumfang von Teleheimarbeit und der formalen oder informellen Nutzung von Teleheimarbeit zur Verfügung standen. Diese hätten ein noch differenziertes Bild ermöglicht.

Trotz der genannten Einschränkungen und abgesehen von den spezifischeren Differenzierungen, ist eine aus unserer Sicht besonders wichtige generelle Schlussfolgerung aus unseren Analysen, dass für die Beurteilung von Teleheimarbeit ein differenzierter, und hier insbesondere zwischen verschiedenen betrieblichen Ungleichheitsregimen unterscheidender Blick unabdingbar ist. Teleheimarbeit wirkt sich nicht per se in die eine oder andere Richtung aus, sondern erst die Einbettung solcher spezifischer Maßnahmen in umfassendere betriebliche Bedingungen - sowohl auf der Ebene der Organisationskulturen als auch der direkten innerbetrieblichen Beziehungen - entscheidet darüber, welche Konsequenzen für die Beschäftigten entstehen. Die alleinige Erfassung der Verfügbarkeit von Teleheimarbeit auf Betriebsebene hätte deshalb auch zu Fehlschlüssen geführt. In den von uns untersuchten Großbetrieben standen in den Betrieben, in denen Teleheimarbeit nicht als Teil betrieblicher Personalpolitik angegeben wurde, diese bis auf eine Ausnahme dennoch in unterschiedlichem Ausmaß informell zur Verfügung. Da die betrieblich dargestellte Verfügbarkeit in den Analysen keinen Einfluss auf geschlechtsspezifische Einkommensungleichheiten hatte, hätte man fälschlicherweise geschlussfolgert, dass die 
Verfügbarkeit von Teleheimarbeit im Betrieb Einkommensungleichheiten zwischen Männern und Frauen, unter Berücksichtigung des Vorhandenseins von Kindern, weder reduziert noch verstärkt. In einer Situation, in der Teleheimarbeit im politischen Raum kontrovers diskutiert wird, allerdings pauschalierend auf dem Niveau von Pro oder Contra, Schwarz oder Weiß, ist allein schon der Verweis auf die notwendige Differenzierung nach betrieblichen Kontexten an sich von großer Bedeutung. Es wäre zu wünschen, dass sich die Forschung weiter in diese Richtung bewegt, sodass unangemessen pauschalierende Bewertungen es in Zukunft schwerer haben.

\section{Literatur}

Abendroth, Anja-Kristin, und Laura Den Dulk. 2011. Support for the work-life balance in Europe: The impact of state, workplace and family support on work-life balance satisfaction. Work, Employment and Society 25:234-256. https://doi.org/10.1177/0950017011398892.

Abendroth, Anja-Kristin, und Mareike Reimann. 2018. Telework and work-family conflict across workplaces: Investigating the implications of work-family-supportive and high-demand workplace cultures. In Contemporary perspectives in family research: The work-family interface: Spillover, complications, and challenges 15:323-348. https://doi.org/10.1108/S1530-353520180000013017.

Abendroth, Anja-Kristin, Silvia Maja Melzer, Peter Jacobebbinghaus und Fabienne Schlechter. 2014. Methodenbericht Beschäftigten- und Partnerbefragung des Linked-Employer-Employee Panels (LEEPB3) Projekt B3: „Wechselwirkungen zwischen Verwirklichungschancen im Berufs- und Privatleben“ (SFB 882 Technical Report Series. Vol.6.) Bielefeld: DFG Research Center (SFB) 882 From Heterogeneities to Inequalities.

Abendroth, Anja-Kristin, Silvia Maja Melzer, Alexandra Kalev und Donald Tomaskovic-Devey. 2017. Women at work: Women's access to power and the Gender Earning Gap. ILR Review 70:190-222. https://doi.org/10.1177/0019793916668530.

Achatz, Juliane. 2005. Geschlechtersegregation im Arbeitsmarkt. In Arbeitsmarktsoziologie. Probleme, Theorien, empirische Befunde, Hrsg. Martin Abraham und Thomas Hinz, 263-301. Wiesbaden: VS Verlag für Sozialwissenschaften.

Acker, Joan. 2006. Inequality regimes: Gender, class, and race in organizations. Gender \& Society 20:441-464. https://doi.org/10.1177/0891243206289499.

Allen, Tammy D., Timothy D. Golden und Kirsten Shockley. 2015. How effective is telecommuting? Assessing the status of our scientific findings. Psychological Science in the Public Interest 16:40-68. https://doi.org/10.1177/1529100615593273.

Allison, Paul D. 2005. Fixed effects regression methods for longitudinal data using SAS. Cary, NC.: SAS Press.

Almer, Elizabeth D., Jeffrey R. Cohen und Louise E. Single. 2004. Is it the kids or the schedule? The incremental effect of families and flexible scheduling on perceived career success. Journal of Business Ethics 54:51-65. https://doi.org/10.1023/B:BUSI.0000043496.47644.38.

Baltes, Boris B., Thomas B. Briggs, Josph W. Huff, Julie A. Wright und George A. Neuman.1999. Flexible and compressed work week schedules: A meta-analysis of their effects on work-related criteria. Journal of Applied Psychology 84:496-513.

Blair-Loy, Mary, und Amy S. Wharton. 2002. Employees' use of work-family policies and the workplace social context. Social Forces 80:813-845. https://doi.org/10.1353/sof.2002.0002.

Brenke, Karl, 2016. Home Office: Möglichkeiten werden bei weitem nicht ausgeschöpft. DIW Wochenbericht $5 / 2016$.

Brunsson, Nils. 2003. Organised hypocrisy. In The Northern Lights: Organization theory in Scandinavia. Hrsg. Barbara Czarniawska und Guje Sevón, 201-215. Copenhagen: Copenhagen Business School Press.

Byron, Kristin. 2005. A meta-analytic review of work-family conflict and its antecedents.

Castilla, Emilio. 2008. Gender, race, and meritocracy in organizational careers. American Journal of Sociology 113:1479-1526. https://doi.org/10.1086/588738.

Cha, Youngjoo, und Kim A. Weeden. 2014. Overwork and the slow convergence in the gender wage gaps. American Sociological Review 79:457-484. https://doi.org/10.1177/0003122414528936. 
Chung, Heeejung. 2017a. National-level family policies and worker's access to schedule control in a European comparative perspective: Crowding out or in, and for whom? Journal of Comparative Policy Analysis: Research and Practice. https://doi.org/10.1080/13876988.2017.1353745.

Chung, Heejung. 2017b. 'Women's work penalty' in access to flexible working arrangements across Europe. European Journal of Industrial Relations. https://doi.org/10.1177/0959680117752829.

Chung, Heejung, und Mariska Van der Horst. 2018. Flexible working and unpaid overtime in the UK: The role of gender, parental and occupational status. Social Indicators Research. https://doi.org/10.1007/ s11205-018-2028-7.

Chung, Heejung, und Tanja Van der Lippe. 2018. Flexible working, work-life balance, and gender equality: Introduction. Social Indicator Research 1-17. https://doi.org/10.1007/s11205-018-2025-x.

Correll, Shelley J., Stephen Benard und In Paik. 2007. Getting a job: Is there a motherhood penalty? American Journal of Sociology 112:1297-1338. https://doi.org/10.1086/511799.

Coyle-Shapiro, Jacqueline A.-M. und Neil Conway. 2004. The employment relationship through the lens of socal exchange. In The Employment Relationship: Examining Psychological and Contextual Perspectives, Hrsg. Jacqueline A.-M. Coyle-Shapiro, Linn M. Shore, Susan M. Taylor und Lois Tetrick, 5-28. Oxford, U. K.: Oxford University Press.

Cropanzano, Russell, und Marie S. Mitchell. 2005. Social exchange theory: An interdisciplinary review. Journal of Management 31:874-900. https://doi.org/10.1177/0149206305279602.

Deitch, Cynthia H., und Matt L. Huffman. 2001. Family responsive benefits and the two-tiered labor market. In Working families: The transformation of the american home, Hrsg. Rosanna Hertz und Nancy L. Marshall, 103-130. Berkeley, CA: University of California Press.

Demerouti, Evangelia, Arnold Bakker, Friedhelm Nachreiner und Wilmar B. Schaufeli. 2001. The job demands-resources model of burnout. Journal of Applied Psychology 86:499-512. https://doi.org/10. 1037/0021-9010.86.3.499.

Diewald, Martin, und Thomas Faist. 2011. Von Heterogenitäten zu Ungleichheiten: Soziale Mechanismen als Erklärungsansatz der Genese sozialer Ungleichheiten. Berliner Journal für Soziologie 21:91-114.

Diewald, Martin, Reinhard Schunck, Anja-Kristin Abendroth, Silvia Maja Melzer, Stephanie Pausch, Mareike Reimann und Björn Andernach. 2015. The Sfb882-B3 linked employer-employee panel survey (LEEP-B3). Schmollers Jahrbuch 134:379-389.

Dobbin, Frank, Daniel Schrage und Alexandra Kalev. 2015. Rage against the iron cage: The varied effects of bureaucratic personnel reforms on diversity. American Sociological Review 80:1014-44.

Den Dulk, Laura. 2001. Work-family arrangements in organisations: A cross-national study in the Netherlands, Italy, the United Kingdom and Sweden. Amsterdam: Rozenberg.

Den Dulk, Laura, und Judith De Ruijter. 2008. Managing work-life policies: Disruption versus dependency arguments. Explaining managerial attitudes towards employee utilization of work-life policies. The International Journal of Human Resource Management 19:1222-1236. https://doi.org/10.1080/ 09585190802109986.

Den Dulk, Laura, Pascale Peters und Erik Poutsma. 2012. Variations in adoption of workplace work-family arrangements in Europe. The International Journal of Human Resource Management 23:2785-2808. https://doi.org/10.1080/09585192.2012.676925.

England, Paula. 1994. Neoclassical economists' theories of discrimination. In Equal employment opportunity: Labor market discrimination and public Policy, Hrsg. Paul Burstein, 59-60. New York: Aldine de Gruyter.

England, Paula. 2010. The gender revolution: Uneven and stalled. Gender and Society 24:149-66. https:// doi.org/10.1177/0891243210361475.

England, Paula, George Farkas, Barbara S. Kilbourne und Thomas Dou. 1988. Explaining occupational sex segregation and wages: Findings from a model with fixed effects. American Sociological Review 53:544-558.

Filer, Randall K. 1985. Male-female wage differences: The importance of compensating differentials. Industrial and Labor Relations Review 38:426-437.

Gajendran, Ravi. S., und David A. Harrison. 2007. The good, the bad, and the unknown about telecommuting: Meta-analysis of psychological mediators and individual consequences. Journal of Applied Psychology 92:1524-1541. https://doi.org/10.4119/unibi/sfb882.2014.12.

Gambles, Richenda, Suzan Lewis und Rhona Rapoport. 2006. The myth of work-life balance. Chichester: John Wiley.

Glass, Jennifer. 2004. Blessing or curse? Work-family policies and mother's wage growth over time. Work and occupations the daddy bonus? Organizational hegemonic masculinity and the impact of fatherhood on earnings. Gender \& Society 24:717-745. https://doi.org/10.1177/089124321038672. 
Goffman, Erving. 1963. Stigma: Notes on the management of spoiled identy. Englewood Cliffs, N.J: Prentice-Hall.

Hodges, Melissa J., und Michelle J. Budig. 2016. Who gets an examination of women, men, loneparents, and parents with partners. Journal of Organizational Behavior 33:1095-1119.

Initiative D21 e. V.2019. D21 Digital Index 2018/19. Jährliches Lagebild zur Digitalen Gesellschaft. StobaDruck. https://initiatived21.de/app/uploads/2018/01/d21-digital-index_2017_2018.pdf(Zugegriffen: 04.03.2019).

Kelliher, Clare, und Deirdre Anderson. 2010. Doing more with less? Flexible working practices and the intensification of work. Human Relations 63:83-106.

Kleemann, Frank. 2005. Die Wirklichkeit der Teleheimarbeit. Berlin: Ed. Sigma.

Konrad, Alison M., und Yang Yang. 2012. Is using work-life interface benefits a career-limiting move? Journal of Organization Behavior 31:367-394. https://doi.org/10.1177/0730888404266364.

Kossek, Ellen. E. 2016. Managing work-life boundaries in the digital age. Organizational Dynamics 45:258-270. https://doi.org/10.1016/j.orgdyn.2016.07.010.

Kossek, Ellen E., Suzan Lewis und Leslie B. Hammer. 2010. Work-life initiatives and organizational change: Overcoming mixed messages to move from the margin to the mainstream. Human Relation 63:3-19. https://doi.org/10.1177/0018726709352385.

Lott, Yvonne. 2015. Working time flexibility and autonomy. A European perspective. European Journal of Industrial Relations 21:259-274. https://doi.org/10.1177/0959680114543604.

Lott, Yvonne. 2018. Does flexibility help employees switch off from work? Flexible working-time arrangements and cognitive work-to-home spillover for women and men in Germany. Social Indicators Research 1-24. https://doi.org/10.1007/s11205-018-2031-z.

Lott, Yvonne. 2019. Weniger Arbeit, mehr Freizeit? Wofür Mütter und Väter flexible Arbeitsarrangements nutzen. WSI Report Nr. 47.

Lott, Yvonne, und Heejung Chung, H. 2016. Gender discrepancies in the outcomes of schedule control on overtime hours and income in Germany. European Sociological Review 32:752-765. https://doi.org/ 10.1093/esr/jcw032.

McDonald, Paula K., Kerry Brown und Lisa M. Bradley. 2005. Explanations for the provision-utilisation gap in work-life policy. Women in Management Review 20:37-55. https://doi.org/10.1108/ 09649420510579568

Oakley, Anee. 2000. Experiments in knowing: Gender and method in the social sciences. Cambridge:Polity Press.

Pausch, Stephanie, Julia Harand, Reinhard Schunck und Peter Jacobebbinghaus. 2013. Methodenbericht Betriebsbefragung Projekt B3: Wechselwirkungen zwischen Verwirklichungschancen im Berufs-und Privatleben (SFB 882 Technical Report Series, 2). Bielefeld: DFG Research Center (SFB).

Petersen, Trond, Andrew M. Penner und Geir Høgsnes. 2011. The male marital wage premium: Sorting vs. differential pay. ILR Review 64:283-304. https://doi.org/10.1177/001979391106400204.

Phelps, Edmund S. 1972. The statistical theory of racism and sexism The American Economic Review 62:659-661.

Pleck, Joseph H. 1993. Are "family-supportive" employer policies relevant to men? In Men, work, and family, Hrsg. Jane C. Hood, 217-237. Thousand Oaks: SAGE.

Reskin, Barbara F. 2000. The proximate causes of employment discrimination. Contemporary Sociology 29:319-328

Ridgeway, Cecilia L., und Shelley Correll. 2004. Unpacking the gender system: A theoretical perspective on gender beliefs and social relations. Gender \& Society 18:510-531.

Risman, Barbara J. 2014. Gender as a social structure. Gender \& Society 18:429-450. https://doi.org/10. $1177 / 0891243204265349$.

Schaufeli, Wilmar, und Arnold B. Bakker. 2004. Job demands, job resources, and their relationship with burnout and engagement. Journal of Organizational Behavior 25:293-315.

Spence, Michael. 1973. Job market signaling. Quarterly Journal of Economics 87:355-374.

Swanberg, Jennifer E., Marcie Pitt-Catsouphes und Krista Drescher-Burker. 2005. A question of justice: Disparities in employees' access to flexible schedule arrangements. Journal of Family Issues 6:866-895. https://doi.org/10.1177/0192513X05277554.

Tilly, Charles. 1998. Durable inequality. Berkeley: University of California Press.

Tomaskovic-Devey, Donald. 2014. The relational generation of workplace inequalities. Social Currents 1:51-73. Https://doi.1177/2329496513514032.

Tomaskovic-Devey, Donald, und Dustin Avent-Holt. 2019. Relational inequalities: An organizational approach. Oxford: Oxford University. 
Van der Lippe, Tanja, und Zoltán Lippényi. 2018. Beyond formal access: Organizational context, working from home, and work-family conflict of men and women in European workplaces. Social Indicators. https://doi.org/10.1007/s11205-018-1993-1.

Van der Lippe, Tanja, Leonie Van Breeschoten, und Margriet Vam Hek. 2018. Organizational work-life policies and the gender wage gap in European workplaces. Work and Occupations. https://doi.org/10. 1177/0730888418791652.

Williams, Joan, Blair-Loy, Mary, und Berdahl, Jennifer. L. 2013. Cultural schemas, social class, and the flexibility stigma. Journal of Social Issues 69:209-234. 